\title{
Effect of vertical ground motions on horizontal response of structures
}

\author{
N. N. Ambraseys and J. Douglas \\ Department of Civil and Environmental Engineering, \\ Imperial College of Science, Technology and Medicine, \\ London, \\ SW7 2BU, U.K. \\ Tel: +44 (0)20 75946059 \\ Fax: +44 (0)20 72252716 \\ Email: n.ambraseys@ic.ac.uk
}




\begin{abstract}
Single-degree-of-freedom (SDOF) elastic models are commonly used for gaining an understanding of the response of structures to earthquake ground motions. The standard SDOF model used does not account for the effect of gravity or the combined effect of horizontal and vertical excitations on horizontal response. The purpose of this paper is to review previous work on this topic and to investigate a series of SDOF models that do incorporate these effects and to compare their response to the response of the standard model using 186 strong-motion records of near-field earthquake ground motions. It is found that for most realistic SDOF models and most earthquake ground motions the effect of vertical excitation on horizontal response is small.
\end{abstract}

KEY WORDS: strong ground motion; response spectra; vertical motions

\title{
1 Introduction
}

Single-degree-of-freedom (SDOF) elastic models are commonly used for gaining an understanding of the response of structures to earthquake ground motions. The standard SDOF model usually used does not account for the effect of gravity or the combined effect of horizontal and vertical excitations on horizontal response. There are a series of SDOF models in the literature that do include these effects, however they have not been thoroughly investigated in the past. Therefore the purpose of this paper is a more thorough examination of these models than has been undertaken before.

In this first section we introduce the SDOF models under investigation and review previous work on these models. In later sections we study the different types of response of these models using a large set of near-field earthquake ground motion records and compare their response to the response of the standard model.

\subsection{Standard model}

Consider the SDOF system illustrated in Figure 1. This system consists of a mass $m$ driven by a horizontal ground motion with acceleration $U_{t t}$, with a spring with stiffness $k$ and a dashpot with a coefficient of viscous damping $c$. Let $u(t)$ be the horizontal displacement of the mass at time $t$. Then using Newton's second law and resolving forces horizontally gives:

$$
m u_{t t}+c u_{t}+k u+m U_{t t}=0
$$

Dividing by $m$ and letting $\omega_{0}^{2}=k / m$ and $\xi_{0}=c / 2 \omega_{0} m$ yields the well-known equation of motion: 


$$
u_{t t}+2 \xi_{0} \omega_{0} u_{t}+\omega_{0}^{2} u=-U_{t t}
$$

Equation 1 is usually used to model the response of structures to earthquake excitation, see for example Chopra ${ }^{1}$.

\subsection{Bending models}

Structural models in this section behave as if their supporting beam-column bends; hence their failure mechanism is buckling. From now on these SDOF models will be called bending models.

Consider the SDOF system illustrated in Figure 2(a). This is a massless beam-column, clamped in the ground at the lower end, with a concentrated mass $m$ at the top. When the column is subjected to a horizontal ground acceleration, $U_{t t}$, the linearized equation of motion is ${ }^{2}$ :

$$
u_{t t}+2 \xi_{0} \omega_{0} u_{t}+\omega_{0}^{2}\left(1-\frac{m g}{P_{c r}}\right) u=-U_{t t} .
$$

where $P_{c r}$ is the critical load of the column. Letting $\omega_{1}^{2}=\omega_{0}^{2}(1-\gamma)$ and $\xi_{1}=\xi_{0} \omega_{0} / \omega_{1}$ where $\gamma=$ $m g / P_{c r}$ then have:

$$
u_{t t}+2 \xi_{1} \omega_{1} u_{t}+\omega_{1}^{2} u=-U_{t t}
$$

As can be seen by comparing Equation 1 with Equation 3 gravity loads simply change the natural period of the system and do not alter the amplitude of the response (as long as the correct period is considered).

This derivation shows one problem with using Equation 1 to characterise the response of structures to horizontal seismic loading. If a fundamental period of the structure is found, by using a vibration generator for instance, the structure behaves as if it is in a non-zero gravity field. Consequently the period, $T_{1}=2 \pi / \omega_{1}$, and coefficient of critical damping, $\xi_{1}$, found are non-zero gravity values. A design spectrum, constructed using Equation 1, should be consulted for the corresponding zero-gravity period, $T_{0}=2 \pi / \omega_{0}$, and damping, $\xi_{0}$, and not for the non-zero gravity values. Thus $T_{1}$ and $\xi_{1}$ should be converted using $T_{0}=T_{1} \sqrt{1-\gamma}$ and $\xi_{0}=\xi_{1} \sqrt{1-\gamma}$.

In practice though, since the concept of fundamental period and coefficient of critical damping for a complicated structure are already approximations, failure to use the theoretically correct period and damping coefficient is not serious. For $\gamma=0.33$, i.e. a factor of safety of three, $T_{0}=0.8 T_{1}$ and $\xi_{0}=0.8 \xi_{1}$ thus the discrepancy is not large. As shown below the change of fundamental period due to gravity is not the same for bending and hinging models, further complicating the situation. 
Consider the SDOF system illustrated in Figure 2(b). This model assumes that the structure is infinitely stiff vertically so that vertical displacements due to ground motion are constant throughout the whole column therefore the vertical ground motion is the vertical input into the mass at the top of the column, i.e. it is unaffected by the column.

The derivation of the equation of motion for the system depicted in Figure 2(b) follows the derivation for when vertical ground motion is neglected, but Equation 2 becomes:

$$
u_{t t}+2 \xi_{0} \omega_{0} u_{t}+\omega_{0}^{2}\left(1-\frac{m\left(g+V_{t t}\right)}{P_{c r}}\right) u=-U_{t t}
$$

where $V_{t t}$ is the vertical acceleration (positive downwards). Using the same transformations of variables as before and $\beta=\gamma / g(1-\gamma)$ the equation of motion becomes:

$$
u_{t t}+2 \xi_{1} \omega_{1} u_{t}+\omega_{1}^{2}\left(1-\beta V_{t t}\right) u=-U_{t t}
$$

This model has been studied by a handful of authors to assess the effect of vertical accelerations on the amplitude of the response for elastic systems, namely Lin and $\mathrm{Shih}^{2}$, Orabi and $\mathrm{Ahmadi}^{3}$, Loh and $\mathrm{Ma}^{4}$ and Şafak ${ }^{5}$.

Lin and Shih ${ }^{2}$ use simulated accelerograms of Gaussian white noise modulated by an envelope function using dimensionless variables, through Fokker-Planck equations, in order to compute the expected response. They mention that parametric resonance (see below) is possible but that because earthquakes have short durations, such effects will not cause instability. They show that the correlation between vertical and horizontal ground accelerations only has an effect on the structural response if the structure is not initially at rest, hence this correlation can be ignored.

Although the use of dimensionless variables in Lin and $\mathrm{Shih}^{2}$ leads to a generalised method for characterising the response of SDOF systems governed by Equation 4 it means that the results displayed are not readily useable. They require transformations using realistic structural parameters, such as length of column, natural period and load ratio, before the results can be used for design. Use of Gaussian white noise to simulate the response of SDOF systems to earthquake strong motion is well established, see for example Clough and Penzien ${ }^{6}$. Bycroft ${ }^{7}$ showed that it can be used to derive response spectra which match quite well those from recorded accelerograms. The choices of power spectral density, $\Phi_{11}=0.0220$ and 0.0314 and $\Phi_{22}=0.0141$ and 0.0201 , made in Lin and $S_{\text {Shih }}^{2}$ are unrealistically high . Transforming these dimensionless power spectral densities into $\mathrm{m}^{2} \mathrm{~s}^{-3}$ yields: $\phi_{11}=l \omega_{1}^{2}(1-\gamma) \Phi_{11}$ and $\phi_{22}=\pi^{2} l \omega_{1}^{2}(1-\gamma) \Phi_{22} / 12$. For realistic values of $\omega_{1}, l$ and $\gamma \phi_{11} \gg \Phi_{11}$ and $\phi_{22} \gg \Phi_{22}$. In Liu and Jhaveri ${ }^{8}$ the power spectral densities given are all less than about $0.009 \mathrm{~m}^{2} \mathrm{~s}^{-3}$ and in Orabi and Ahmadi ${ }^{3}$ $0.005544 \mathrm{~m}^{2} \mathrm{~s}^{-3}$ is given as the power spectral density of the NS component of the El Centro record (from 
the El Centro earthquake, 19/5/1940). Therefore $\Phi_{11}$ and $\Phi_{22}$ are much too high. This means that valid conclusions cannot be drawn from their numerical examples. Lin $^{9}$ noted that the numerical examples given may be unrealistic although the theory is correct. Bycroft ${ }^{7}$ originally proposed the use of white noise due to the dearth of actual recordings close to the source of large earthquakes. Today there are many near-field recordings and these can be used rather than simulating strong motion through white noise.

Orabi and $\mathrm{Ahmadi}^{3}$ also use simulated accelerograms of Gaussian white noise modulated using an envelope function and Fokker-Planck equations to evaluate the stochastic response. Also they perform Monte-Carlo simulations directly using segments of white noise in order to check the results. They base the white noise used on the intensities of the NS and vertical components of the El Centro record. The similarity between Monte-Carlo and results using the Fokker-Planck equations is noted for two envelope functions: a constant function (stationary analysis) and an exponential envelope function (nonstationary analysis). Both methods show an increase in response for large load ratios and larger increases for smaller damping ratios. For example for the stationary analysis with $\xi_{1}=0.02$ and $T_{1}=6.3 \mathrm{~s}$ the increase in root-mean-square displacement response as $\gamma$ increases from 0.5 to 0.9 is about $5 \%$ but the increase in response from $\gamma=0.90$ to 0.95 is about $20 \%$. For the same period but with $\xi=0.20$ the corresponding increases are $2 \%$ and $7 \%$. This shows the important influence of damping and load ratio on the effect of vertical excitations for this model. They also find the relative velocity response spectrum of the El Centro $\mathrm{N}-\mathrm{S}$ record with and without vertical excitation for different load ratios. They note the similarity between their theoretical results and the computed spectra, their conclusions on the importance of damping and load ratio also hold for this accelerogram.

The study of Orabi and Ahmadi ${ }^{3}$ has a number of limitations. Their results rely on white noise with simple envelope functions to represent the horizontal and vertical ground accelerations which may not model all the characteristics of recorded earthquake strong motion. They also base their input ground intensities on the El Centro record which is no longer one of the most intense ground motions available, thus their results underestimate how much the vertical ground motion may amplify the horizontal response.

Şafak $^{5}$ uses four near-field records, three from the Kocaeli earthquake $\left(17 / 8 / 1999, M_{w}=7.4\right)$ (Yarmica, Izmit and Sakarya) and one from the Düzce earthquake (12/11/1999, $M_{w}=7.1$ ) (Düzce), to investigate the response of structures governed by Equation 4. Four different load ratios are used, $\gamma=0,0.2,0.4$ and 0.6 and the displacement response spectra for $5 \%$ damping for these different $\gamma$ values are plotted for each fault-normal record. It is found that at long periods, $T=8.0 \mathrm{~s}$, the spectral displacements from the Sakarya record are 2.5 times higher for $\gamma=0.6$ than for $\gamma=0$, which Şafak ${ }^{5}$ 
suggests is because the amplitudes of the vertical and horizontal accelerations of similar size and that this record has more long-period energy that those from other stations.

The main problem with the analysis of Şafak ${ }^{5}$ is that the displacement spectra are plotted in terms of the non-zero gravity period and damping (see above) therefore the differences found are almost entirely due to the effect of gravity on the natural period and damping and not because of the vertical ground motion. Plotting the displacement spectra in terms of the non-zero gravity parameters makes it almost impossible to distinguish the effect of the vertical excitation from the effect of gravity.

Loh and $\mathrm{Ma}^{4}$ is the only known published study of the response of SDOF systems governed by Equation 4 which uses a large number of actual strong-motion records. Two parameters are mentioned as important: the load ratio, $\gamma$, and the size of ratio between horizontal and vertical PGA. Thirty ${ }^{1}$ Taiwanese records from a hard site are used to develop a uniform hazard response spectrum. Both the horizontal and vertical accelerograms were normalised to have a PGA of $1 \mathrm{~g}$ and $\gamma=0.5$ was used (it was noted that larger values of $\gamma$ caused instability although the reason is not given, see below) and uniform hazard response spectra were computed which have the same probability of being exceeded at all periods. These can then be scaled by the design level PGA to yield a design spectrum. They conclude that for $5 \%$ damping, $\gamma=0.5$ and horizontal and vertical PGA normalised to $1 \mathrm{~g}$ vertical excitation increases the response by $33 \%$ compared with when only horizontal excitation is considered.

Loh and $\mathrm{Ma}^{4}$ assume that the importance of vertical ground motion on the response of systems governed by Equation 4 is only dependent on PGA and not the other factors known to influence ground motion, e.g. magnitude, distance and local site conditions. It also is based on a vertical to horizontal PGA ratio of unity which is larger than other studies have found. Therefore it may overestimate the importance of vertical acceleration on bending SDOF systems although the authors do mention that a different choice of this ratio may affect the results (see for example their Figure 12). Also $\gamma=0.5$ is a higher load ratio than imposed on most buildings.

Inelastic systems based on Equation 4 but with a non-linear force-displacement term have been investigated by Shih and $\operatorname{Lin}^{10}$. Following on from Lin and Shih ${ }^{2}$ they define their equation of motion in terms of nondimensional quantities (although the nondimensional quantities are slightly different to those in Lin and $\mathrm{Shih}^{2}$ ) which again makes the use of their results difficult. Material non-linearity of the structure is modelled using a function proposed by Hata and Shibata, which is a simple hysteretic function with one parameter, $0 \leq r<1$, which controls the non-linearity of the system (the system was assumed to have yielded from the beginning). The ground accelerations are modelled as amplitude modulated Gaussian white noise processes and the expected response is found through Fokker-Planck equations (also

\footnotetext{
${ }^{1}$ The caption of their Figure 7 says fifty records were used.
} 
used in Lin and Shih ${ }^{2}$ although complications arise due to the non-linearity of the system). Numerical results for two different values of $r, 0.1$ and 0.5 , and two levels of spectral density are presented. As for Lin and Shih ${ }^{2}$ the spectral densities chosen, $2 \pi \Phi_{11}=1,2$ and 3 and $2 \pi \Phi_{22}=0.64 \Phi_{11}$ are much too large for earthquake excitation therefore the numerical results are not valid. They do find though (which is probably not dependent on the incorrect spectral densities they use) that one hysteretic system can behave very differently from another system when gravity and vertical accelerations are included. Thus the results are more sensitive to model parameters than is so for linear elastic models.

Consider the SDOF system illustrated in Figure 2(c). This model assumes that the structure has finite stiffness vertically and that vertically the column responds like a SDOF system governed by an equation of motion like Equation 1 although not necessarily with the same damping and natural period as in the horizontal direction. This means that the system is separable into the response vertically and the response horizontally which is affected by the vertical response but not vice versa.

The equation of motion of this system is governed by:

$$
u_{t t}+2 \xi_{1} \omega_{1} u_{t}+\omega_{1}^{2}\left(1-\beta u_{t t}^{v}\right) u=-U_{t t},
$$

where $u_{t t}^{v}=u_{t t}^{v}\left(t, T_{V}, \xi_{V}\right)$ is the vertical response acceleration for vertical natural period, $T_{V}$, and damping $\xi_{V}$, i.e. $u_{t t}$ from Equation 1 for $\omega_{0}=2 \pi / T_{V}, \xi_{0}=\xi_{V}$ and input acceleration, $V_{t t}(t)$.

\subsection{Hinging models}

Structural models in this section behave as if their supporting beam-column is hinged at the base. From now on these SDOF models will be called hinging models. Consider an inverted pendulum with an elastic hinge at the base (Figure 3(a)). The equation of motion of this system is:

$$
u_{t t}+2 \xi_{0} \omega_{0} u_{t}+\left(\omega_{0}^{2}-g / l\right) u=-U_{t t} .
$$

As for the bending case a transformation of variables is useful. If $\omega_{0}^{2}>g / l$ (if this does not hold the system does not oscillate but is unstable) then letting $\omega_{1}=\sqrt{\omega_{0}^{2}-g / l}$ and $\xi_{1}=\xi_{0} \omega_{0} / \omega_{1}$ :

$$
u_{t t}+2 \xi_{1} \omega_{1} u_{t}+\omega_{1}^{2} u=-U_{t t}
$$

Requiring $\omega_{0}^{2}>g / l$ gives a limit, i.e. $l>m g / k$, on the smallest $l$ can be for the pendulum simply to withstand gravity loads and so all structures must satisfy this condition, even if they are not designed for earthquake loads. 
As for the bending model, a design spectrum constructed using equation 1 should be consulted for the corresponding zero-gravity period, $T_{0}$, and damping, $\xi_{0}$, given by $T_{0}=T_{1} / \sqrt{1+T_{1}^{2} g /\left[(2 \pi)^{2} l\right]}$ and $\xi_{0}=\xi_{1} / \sqrt{1+T_{1}^{2} g /\left[(2 \pi)^{2} l\right]}$ where $T_{1}$ and $\xi_{1}$ are non-zero gravity values.

Figure 4 shows the factor, $1 / \sqrt{1+T_{1}^{2} g /\left[(2 \pi)^{2} l\right]}$, against $T_{1}$ for different lengths of pendula, $l$. This shows how much the natural period and damping changes when gravity forces are considered. As can be seen the change in natural period and damping is only large for short columns and long periods.

Jennings and Husid ${ }^{11}$, Husid ${ }^{12}$, Sun et al. ${ }^{13}$, Bernal ${ }^{14}$ and Fenwick et al. ${ }^{15}$ all investigate this model amongst others and conclude the effect of gravity is negligible, which it is if $l$ is reasonably long so that the change in natural period and damping is small.

Jennings and Husid ${ }^{11}$ and Husid ${ }^{12}$ study a model similar to that specified in Equation 7, although not making the assumption that $\theta$ is small ${ }^{2}$, hence their equation of model is slightly more complex, for elastoplastic and bilinear hysteretic structures. They consider many choices of natural period $(0.5,1.0$, 1.5 and $2.0 \mathrm{~s})$, length of pendulum $(1.5,3.0,4.5,6,7.5$ and $9 \mathrm{~m})$ and yield level $(0.05 \mathrm{~g}$ and $0.10 \mathrm{~g})$ each with damping of $2 \%$ of critical. Simulated accelerograms of stationary Gaussian random processes of $60 \mathrm{~s}$ duration are used to investigate the time to collapse of such structures. They find that the time to collapse depends hyperbolically on the ratio of earthquake strength to yield strength, linearly on length of pendulum and is highly dependent on duration (for longer records less intense motion is required for the structure to collapse), but it is independent of natural period. For the bilinear force-deformation relation, if the ratio of the second slope to the initial slope is sufficiently high collapse is prevented. Results are confirmed using actual accelerograms.

Sun et al. ${ }^{13}$ investigate a model similar to that specified in Equation 7 but for a force-displacement curve which has ideal elastoplastic behaviour in extension and buckles at zero load in contraction using phase-plane analysis. They find three equilibrium positions using static methods, conditions for when the system will collapse and will suffer a residual displacement after the shaking has stopped. They use the NS El Centro record to illustrate their results. Two design criteria are proposed based on the conditions required for no large residual displacements and for no collapse, in terms of displacement spectra and input energy.

Bernal ${ }^{14}$ computes amplification factors for gravity effects using four strong-motion records (Olympia S86W, El Centro S00E, Taft S69E and Pacoima Dam S16E) in terms of a dimensionless stability coefficient and ductility factor for elastoplastic systems. A simple limit on the size of the stability coefficient, $\theta=g / \omega_{0}^{2} l$, is given based on earthquake codes. From this the conclusion is drawn that structures in regions of relatively low seismic coefficients, i.e. low design acceleration, are less protected, by the in-

\footnotetext{
${ }^{2}$ They verify that there is little difference between predicted responses when this assumption is made and when it is not made.
} 
terstorey drift limitation, against inelastic gravity effects than those in areas of higher design acceleration. Systems with six ductilities $(1,2,3,4,5$ and 6$)$, nine stability coefficients ( 0 to 0.2$)$ and 37 periods $(0.2$ to $2 \mathrm{~s}$ ) were investigated for each of the records. No significant correlation was found between period and amplification but an expression for predicting amplification due to gravity based on ductility and stability coefficient is given. This expression was found to give different predictions than those given in codes, some of which are shown to under predict amplification.

Fenwick et al. ${ }^{15}$ use a number of strong-motion records, although they base most of their results on an artificial record of about $25 \mathrm{~s}$ duration, to find amplification factors for elastoplastic and bilinear structures. They find that the strain hardening ratio (the ratio between the gradient of the first and second slope of the bilinear force-deformation relation) is not significant for amplification but that viscous damping does make a large difference. They use the Cholame Shandon Array 2W N65E record (from Parkfield earthquake, 28/6/1966), which has a short duration of strong shaking, and compare the amplification factors with those for the El Centro record and the artificial record and find they are much lower. Hence duration has a large effect. They also find that for some records amplifications are not independent of period over its entire period range. Equations are given for amplification factors in terms of ductility and period for firm and flexible subsoils.

Consider the SDOF system illustrated in Figure 3(b). The derivation of the equation of motion for this system follows that given in above but Equation 6 becomes (since vertical ground acceleration, $V_{t t}$, acts like an additional gravity force):

$$
u_{t t}+2 \xi_{0} \omega_{0} u_{t}+\left(\omega_{0}^{2}-\left(g+V_{t t}\right) / l\right) u=-U_{t t}
$$

Defining $\omega_{1}$ and $\xi_{1}$ as before and letting $\beta=1 /\left(\omega_{0}^{2} l-g\right)=1 / \omega_{1}^{2} l$ yields equation 4 again but with different $\xi_{1}, \omega_{1}$ and $\beta$.

Jennings and Husid ${ }^{11}$ and Husid ${ }^{12}$ as part of their studies also apply vertical ground motion as well as gravity loads and horizontal motion and find that vertical ground motion is relatively unimportant in controlling the time to collapse.

Consider the SDOF system illustrated in Figure 3(c). As for the bending case, see Figure 2(c), this model assumes that the structure is finitely stiff vertically and that vertically the column responds like a SDOF system governed by an equation of motion like Equation 1 although with not necessarily the same damping and natural period as in the horizontal direction. This means that the system is separable into the response vertically and the response horizontally which is affected by the vertical response but not vice versa. The equation of motion of this system is Equation 5 again but with different $\xi_{1}, \omega_{1}$ and $\beta$.

Tani and Soda ${ }^{16}$ present an investigation using a similar model to Equation 5 although using a bi- 
linear force-displacement relationship, with positive stiffness ratio, similar to that used by Shih and $\operatorname{Lin}^{10}$. They assume the structure continues to behave elastically in the vertical direction even when plastic deformation takes place in the horizontal direction. Horizontal ground motion is assumed to be quasi-nonstationary white noise and vertical excitation is stationary white noise. Statistical mean square response of the system is expressed by a Voltera type integral equation and solved using Laplace transforms. An equivalent linearization method is used to model the bilinear hysteresis which they find to be accurate. Numerical results are given for ten models with different stiffness ratios, $r$, heights, $H$, yield displacements and natural periods and for the three conditions: horizontal excitation only, horizontal and gravity loads and horizontal and vertical excitation and gravity loads. All of their models have vertical natural periods equal to a tenth of the the horizontal period, vertical damping equal to $10 \%$ and horizontal damping equal to $2 \%$. They conclude that gravity loads can be important, increasing the displacement more than $10 \%$, for tall structures and especially those with small stiffness ratio. They find vertical excitation can be ignored due to its small effect.

The study of Tani and Soda ${ }^{16}$ is small scale, only a few models are considered which do not cover different combinations of horizontal and vertical natural period and damping which could occur in structures. They also do not subject their models to particularly large excitations, the PGA of their most intense white noise excitation is $6 \mathrm{~ms}^{-2}$ and the power spectral density of the vertical excitation of all of their simulations is a quarter of the horizontal density.

\subsection{Conclusions}

This section shows that although some work has been completed on how vertical ground motion affects structural response, many of these studies are too small scale for their conclusions to be general. Hence there is a need for a more general approach using a range of structural models, structural parameters and ground motion inputs to derive some general conclusions on the importance of vertical ground motion to design.

Many of the SDOF system studies do not base their results on actual strong-motion recordings but on white noise representation of ground shaking. Although white noise representation may yield adequate estimates of the importance of vertical excitation for most earthquake ground motions which occur, Newmark and Rosenblueth ${ }^{17}$, p. 302 state '[a]dditional confirmation of the orders of magnitude of Monte Carlo results should in general be obtained from spotchecks using records of actual earthquakes'. When white noise was first used to simulate strong-motion records, in the 1960s, there were few records of actual earthquakes especially those from the near field of large earthquakes. Now though, there are thousands of strong-motion recordings are available of which a large fraction are from the near field of 
reasonably large earthquakes, thus no longer do studies on the response of structures to simultaneous horizontal and vertical excitation need to be solely based on white noise approximations.

Those studies which do use actual accelerograms often use only a handful and often they only use the El Centro record, although many other records exist which are more reliable (due to better recording and processing) and contain more intense motion. Thus a large suite of time-histories needs to be utilised to give reliable conclusions which are based on ground motions which have actually occurred.

Newmark and Hall ${ }^{18}$ note that it is still difficult to construct mathematical models that lead to satisfactory results and that are not complicated to the point of becoming impractical for analysis of complex structures'. Shih and Lin ${ }^{10}$ noted, specifically for combined horizontal and vertical excitation, that two inelastic SDOF systems can behave very differently under the same seismic action. Therefore an understanding the response of simple models is needed before complex models can be studied. An investigation of these simple models follows.

\section{Data used}

We selected 186 free-field, chiefly triaxial strong-motion records from 42 earthquakes using the criteria: $M_{s} \geq 5.8$, distance to surface projection of rupture $d \leq 15 \mathrm{~km}$ and focal depth $h \leq 20 \mathrm{~km}$. Because of space limitations those chosen records and other tabulated material are listed in Ambraseys and Douglas ${ }^{19}$. The majority $(72 \%)$ came from western North America, the rest from Europe and from other parts of the world. Their distribution with earthquake mechanism is: 98 or $53 \%$ thrust, 72 or $39 \%$ strike-slip and 16 or $9 \%$ normal. For more details see Ambraseys and Douglas ${ }^{19}$ and Ambraseys and Douglas ${ }^{20}$.

\section{Stability}

\subsection{Bending model}

Consider the homogenous Equation 4, i.e. $U_{t t}=0$, and let $\alpha=\left(1-\beta V_{t t}\right)$ be constant for a given period of time. Then looking for solutions of Equation 4 of the form $u=K e^{p t}$ leads to the equation:

$$
p=\omega_{1}\left(-\xi_{1} \pm \sqrt{\xi_{1}^{2}-\alpha}\right) .
$$

Solutions of Equations $1,3 \& 7$ correspond to $\alpha=1, \omega_{1}$ replaced by $\omega_{0}$ and $\xi_{1}$ replaced by $\xi_{0}$ and for both solutions $p$ has a negative real part therefore the amplitude of the motion decays with time. This is not so in equation 9. If $\xi_{1}^{2}-\alpha>0$ and $\sqrt{\xi_{1}^{2}-\alpha}>\xi_{1}$ then one solution of equation 9 will be positive. This means that one of the solutions of equation 4 has the form $u=K e^{p_{+} t}$ where $p_{+}>0$, a solution 
which rapidly tends to $\infty$ as $t \rightarrow \infty$. Therefore if $\beta V_{t t}>1$ for a reasonable length of time then the displacements of the mass can become very large. This inequality is equivalent to:

$$
\begin{aligned}
V_{t t} & >\frac{g(1-\gamma)}{\gamma} \\
\text { or } \gamma & >\frac{1}{V_{t t} / g+1}
\end{aligned}
$$

Once the column is displaced horizontally from the vertical gravity and positive vertical accelerations mean it is easier for displacements of the mass horizontally to continue. As the displacement increases the equivalent stiffness of the column decreases and so the mass continues to be deflected by more and more. Only the application of a large negative vertical acceleration will counteract this process. Inequality 10 simply means that bending model cannot withstand forces (gravity plus vertical ground accelerations) greater than its Euler buckling load, $P_{c r}$ if they are sustained for a significant length of time. Note that this upper limit on $\gamma$ holds for all non-zero horizontal input motion.

\subsubsection{Infinite vertical stiffness}

All the records, with vertical components, in the dataset were used to study the onset of instability for increasing $\gamma$. The response spectrum, for 0,2,5,10 and 20\% damping, of each horizontal component was calculated for $\gamma$ between 0 and the $\gamma$ which yields SA $>1000 \mathrm{~ms}^{-2}$ at one or more periods, increasing in 0.01 unit intervals, or $\gamma>0.96^{3}$. From each spectrum the largest $\mathrm{SA}$ for any period, $\operatorname{SA}_{\max }(\gamma)$ was found. Figure 5 shows $\mathrm{SA}_{\max }(\gamma) / \mathrm{SA}_{\max }(\gamma=0)$, i.e. amplification in response due to vertical excitation, against $\gamma$ for one component (for $5 \%$ damping). The dashed line marks the boundary between values of $\gamma$ where the system is stable and $\gamma$ where Inequality 10 holds $(\gamma=0.58$ for this record), i.e. the system could be unstable if the amplitude of vertical acceleration was sustained.

Figure 5 shows that only for values of $\gamma$ close to the region of instability, around $\gamma>0.4$, does SA significantly increase. It also shows that SA of damped systems do not become unrealistically large, i.e. high amplifications, until $\gamma$ is slightly larger, about $\gamma>0.65$, than the smallest value where Inequality 10 holds. This is because the stability condition of the SDOF system is only violated for a short time and large responses are not able to build up.

For each record it is found that the ratio of critical damping used does not strongly affect the value of $\gamma$ above which instability occurs. For example, for the Tabas N74E component instability occurs when $\gamma$ exceeds $0.50,0.62,0.65,0.68$ and 0.75 for $0,2,5,10$ and $20 \%$ damping respectively (see Figure 5).

\footnotetext{
${ }^{3}$ For $\gamma>0.96$ the time to calculate the response became extremely long even for records with small vertical accelerations.
} 
For each value of $\gamma$ the minimum acceleration (limit acceleration) which satisfies Inequality 10 was calculated and the maximum length of time, in the records, for which accelerations higher than this were sustained. Note this is not the total amount of time for which the recorded acceleration is above a threshold but the maximum interval where accelerations are above a threshold. These intervals are also a function of $\gamma$. To calculate this the records were linearly interpolated. Figure 6 shows $\mathrm{SA}_{\max }(\gamma) / \mathrm{SA}_{\max }(\gamma=0)$ against these calculated intervals for one component for $5 \%$ damping.

Figure 6 shows that the SDOF system is still stable if the interval for which the ground acceleration satisfies Inequality 10 is sufficiently small, less than about $0.025 \mathrm{~s}$, but for longer intervals the system's responses can become extremely large, i.e. it is unstable. All examined records show similar behaviour although the length of the interval, during which the ground acceleration is above the limit acceleration, required for instability to occur varies (Table 1). The interval lengths vary because the vertical ground acceleration is a dynamic force and not static and also because of the effect of the combination of horizontal and vertical excitation on the system.

As the load ratio, $\gamma$, increases the period of the peak response decreases because vertical ground motion is usually of a higher frequency than the corresponding horizontal ground motion. For load ratios large enough to increase the response significantly (i.e. close to the unstable region or within the unstable region) the horizontal period at which the largest response occurs is usually between 0.1 and $0.2 \mathrm{~s}$ which reflects the high frequency nature of vertical ground motion. Therefore Table 1 shows that instability occurs if the length of the interval when Inequality 10 holds is greater than some fraction (usually about an eighth to a quarter for realistic damping levels) of the horizontal period for which the instability occurs (the most commonly interval given in Table 1 is estimated by a visual inspection of a graph such as Figure 6 with all components plotted). Table 1 shows that damping does not have a strong influence on the onset of instability due to too large a load ratio, $\gamma$.

The analysis shows that the SDOF systems governed by Equation 4 or 5 become unstable, i.e. the response of the system is unphysically large, for earthquake loading when the vertical ground acceleration is above a limit, given by simple Inequality 10 , for longer than between 0 and $0.13 \mathrm{~s}$ and that the ratio of critical damping present in the SDOF system does not have a large influence on this.

The limit on the length of the interval that produces unrealistically large responses is related to the natural horizontal period of the system, $T_{h}$. For systems with extremely short natural horizontal periods $\left(T_{h}<0.1 \mathrm{~s}\right)$ the critical length of interval approaches zero, i.e. if Inequality 10 holds for any length of time during the earthquake then the system will become unstable. For systems with extremely long natural horizontal periods $\left(T_{h}>10 \mathrm{~s}\right)$ then the critical interval tends to the longest interval within the acceleration time-history between zero crossings. This is shown in Table 2 using the Tabas N74E 
component. Due to the correction technique there is little energy in the period range of the records 0 to $0.04 \mathrm{~s}$ and beyond $5 \mathrm{~s}$ thus results for natural horizontal periods within these ranges may be unreliable. Table 2 shows that limits on the critical interval mentioned above hold, i.e. for extremely short periods the critical interval is also short and as period increases so does the critical interval reaching an upper limit equal to the maximum time between zero crossings of acceleration (in this case $0.50 \mathrm{~s}$ ).

This result shows that the breakdown of the SDOF systems governed by Equation 4 or 5 is more likely to occur for short-period than long-period systems because the high vertical acceleration which induces instability only needs to be sustained for an extremely short time. For long period systems the high vertical acceleration needs to be sustained for a longer time but this cannot be longer than the maximum time between zero crossings.

For each record in the near-field set the maximum load ratio, $\gamma$, which can be used without Inequality 10 holding was calculated for both infinite and finite vertical stiffness (for natural vertical periods between 0.1 and $2 \mathrm{~s}$ and 2, 5, 10 and 20\% damping). This was done without considering the time the vertical input acceleration is above the critical level. Figure 7 shows the maximum load ratio against the cumulative total of records for which instability may occur for infinite and finite vertical stiffness.

Figure 7 shows that for load ratios of 0.3 to 0.5 most vertical acceleration time-histories will not induce instability for systems with infinite vertical stiffness. In fact for load ratios less than 0.34 the infinitevertical-stiffness SDOF system will definitely not become unstable for any vertical time-history in the set of records which includes the most intense vertical accelerations yet recorded (Nahanni 1 (Nahanni earthquake, 23/12/1985), vertical PGA $=19.4 \mathrm{~ms}^{-2}$ [2 g]; El Centro 6 (Imperial Valley earthquake, 15/10/1979), vertical PGA $=15.5 \mathrm{~ms}^{-2}$ [1.6 g]; Victoria (Victoria earthquake, 9/6/1980), vertical $\mathrm{PGA}=14.7 \mathrm{~ms}^{-2}[1.5 \mathrm{~g}]$ and Tarzana (Northridge earthquake, 17/1/1994), vertical PGA $=10.3 \mathrm{~ms}^{-2}$ $[1.0 \mathrm{~g}])$ ). It is therefore unlikely that for realistic load ratios vertical acceleration will result in the failure of such systems through instability.

\subsubsection{Finite vertical stiffness}

Figure 7 shows that for systems with finite vertical stiffness a number of vertical time-histories will induce instability for load ratios of 0.3 to 0.5 even for large vertical damping. Figure 7 shows that the maximum load ratio which can be used for an analysis of all the records in the near-field set using the bending model and finite vertical stiffness is about 0.1 for 2 and $5 \%$ damping, for $10 \%$ damping it is about 0.15 and for $20 \%$ damping it is about 0.22 . Thus for certain natural vertical periods and realistic choices of $\gamma(0.3$ to 0.5$)$ the bending SDOF system will yield unrealistically large responses (due to the system breaking down) for some of the near-field records. This precludes a general analysis. 


\subsection{Hinging model}

In the same way that a rough upper limit can be found for the bending equation of motions, a lower limit on $l$ can be found for the hinging equation of motions. Following the same steps as above it can be shown that a large response may occur if:

$$
\begin{aligned}
l & <\frac{V_{t t}}{\omega_{1}^{2}} \\
\text { or: } V_{t t} & >l \omega_{1}^{2} \\
\text { or: } V_{t t} & >l \omega_{0}^{2}-g
\end{aligned}
$$

If this inequality holds then the response of the hinging structure can become large. Since $T_{1}=\frac{2 \pi}{\omega_{1}}$ this means that for structures with long natural periods $l$ has to be large for the structure to remain stable. Inequality 11 is the same constraint as that placed on $l$, during the derivation of the equation of motion when vertical ground acceleration is neglected (see above), modified due to the presence of vertical ground motion.

All the records, with vertical components, in the near-field data set were also used to study the onset of instability for decreasing $l$. The response spectrum, for $0,2,5,10$ and $20 \%$ damping, of each horizontal component was calculated for $l$ between $5 \mathrm{~m}$ (for $l$ this large, and for the period range of interest, the vertical excitation has no effect) and the $l$ which yields SA $>1000 \mathrm{~ms}^{-2}$ at one or more periods, decreasing by a factor of 0.95 each loop. From each spectrum the largest SA for any period, $\mathrm{SA}_{\max }(l)$ was found. Figure 8 shows $\mathrm{SA}_{\max }(l) / \mathrm{SA}_{\max }(l=5 \mathrm{~m})$, i.e. amplification in response due to vertical excitation, against $l$ for one component. Inequality 11 involves frequency (and hence period) thus the boundary between the stable and unstable regions depends on period. The largest period, $2 \mathrm{~s}$, gives the smallest critical $l$ and this is used. In Figure 8 the dashed line marks the boundary between values of $l$ where the system is stable and $l$ where Inequality 11 holds $(l=0.74 \mathrm{~m}$ for this record and natural period of 2 s), i.e. the system could be unstable if the amplitude of the vertical acceleration was sustained.

Figure 8 shows that Inequality 11 is extremely over conservative in its prediction of the stable region, predicting that for $l<0.74 \mathrm{~m}$ stability could be a problem whereas in fact the response only increases for $l<0.07 \mathrm{~m}$ and large responses indicative of instability only occur for $l<0.05 \mathrm{~m}$. The reason for the large difference is that vertical peak ground acceleration is usually associated with high frequency waves which do not affect long period systems which are the ones for which instability is predicted using Inequality 11. Unless a vertical strong motion record contains large amplitude long period accelerations then instability is not a problem for realistic choices of $l$ nor does any amplification due to the vertical 
acceleration occur for realistic $l$ values.

For each record it was found that the ratio of critical damping used did not strongly affect the value of $l$ below which instability occurs. For example, for the Tabas N74E component the value of $l$ below which instability occurs is $0.07,0.06,0.05,0.05$ and $0.04 \mathrm{~m}$ for $0,2,5,10$ and $20 \%$ damping respectively ((see Figure 8).

For each value of $l$ the minimum acceleration (limit acceleration) which satisfies Inequality 11 was calculated and the maximum time the records show sustained accelerations higher than this. Note this is not the total amount of time in the records which the acceleration was above a threshold but the maximum interval where accelerations above a threshold were recorded. Also note that these intervals are a function of $l$ and natural period. To calculate this the records were linearly interpolated. Figure 9 shows $\mathrm{SA}_{\max }(l) / \mathrm{SA}_{\max }(l=5 \mathrm{~m})$ against these calculated intervals for one component for $5 \%$ damping.

Figure 9 shows that the SDOF system is still stable if the interval for which the ground acceleration satisfies Inequality 11 is sufficiently small, less than about $0.2 \mathrm{~s}$, but for intervals longer than a certain length of time the system's responses are extremely large, i.e. it is unstable. All examined records show similar behaviour although there is a range of intervals, during which the ground acceleration is above the limit, required for instability (Table 3). The variation occurs because the vertical ground acceleration is a dynamic force and not static and also because of the effect of the combination of horizontal and vertical excitation on the system. The situation is further complicated because Inequality 11 is a function not only of $l$ but also the natural period of the system.

This analysis shows that the SDOF systems governed by Equation 4 or 5 become unstable, i.e. the response of the system is unphysically large, for earthquake loading when the vertical ground acceleration is above a limit acceleration, given by simple Inequality 11 , for longer than about 0.05 to $1.3 \mathrm{~s}$. Note that the lengths of the column, $l$, for which instability can be a problem $(l<0.07 \mathrm{~m}$ for Tabas N74E component, see Figure 8) are much less than occur in practice especially in long period systems where Inequality 11 may be violated. Also the length of the intervals for which vertical accelerations satisfying Inequality 11 are much longer than those for the bending model. Both these findings mean that large amplification of horizontal response from vertical accelerations is extremely unlikely to occur in practice for structures that can be modelled by the elastic hinging SDOF model.

For each record in the near-field set the minimum length of column, $l$, which can be used before Inequality 11 holds is calculated for both infinite and finite vertical stiffness (for natural vertical periods between 0.1 and $2 \mathrm{~s}$ and 2, 5, 10 and $20 \%$ damping). This was done without considering how long the vertical input acceleration is above the critical level. The calculation for infinite vertical stiffness assumes that the wave associated with vertical PGA is of sufficient period to cause instability in a system with 
natural horizontal period of $2 \mathrm{~s}$. For finite vertical stiffness the vertical spectral acceleration at each period was used to calculate the minimum length of column and then the minimum length from all periods was chosen. Figure 10 shows the minimum length of column against the cumulative total of records for which instability may occur for infinite and finite vertical stiffness.

Figure 10 shows that for columns longer than $2 \mathrm{~m}$ no vertical acceleration time-histories will induce instability for systems with infinite vertical stiffness or finite vertical stiffness. In fact since vertical PGAs are associated with high frequencies the situation for infinite vertical stiffness is much different than Figure 10 suggests. This is because instabilities only occurs if accelerations over the critical level of vertical acceleration are sustained for more than about $0.1 \mathrm{~s}$, as shown above, which will not be so for the wave associated with vertical PGA. Thus this limit on the minimum length of column which can be used is probably much less than $1 \mathrm{~m}$ for both finite and infinite vertical stiffness. The records used for this analysis includes the most intense vertical ground motions yet recorded so this means it is extremely unlikely that hinging systems with a realistic length of column will fail through instability for any vertical acceleration.

\section{Parametric resonance}

For certain combinations of vertical driving frequency, $\Omega_{V}$, and the natural horizontal frequency $\omega_{1}$, systems governed by Equations $4 \& 5$ become dynamically unstable and horizontal vibrations occur; the amplitude of these vibrations rapidly become large. The frequencies at which a system approaches such a resonance (so called parametric resonance) differs from that for ordinary forced vibrations. For sufficiently small values of the longitudinal force this relationship is $\Omega_{V}=2 \omega_{1}{ }^{21}$.

The region of instability can be determined by finding the conditions under which Equations $4 \& 5$ have periodic solutions with period $2 T$. Bolotin ${ }^{21}$ shows that the equations defining the boundary of the unstable region are:

$$
\Omega_{V}=2 \omega_{1} \sqrt{1-2 \xi^{2} \pm \sqrt{4 \xi^{4}-4 \xi^{2}+\left(\frac{A_{V} \beta}{2}\right)^{2}}} .
$$

For $\Omega_{V}=2 \omega_{1}$ this simplifies to $A_{V} \beta=4 \xi$, which is about the largest $A_{V} \beta$ can be before parametric resonance occurs. Figure 11 shows the regions of instability predicted by Equation 12 for different damping levels, $\xi$ against $A_{V} \beta$.

Although parametric resonance is important for periodic horizontal and vertical excitations of long durations whether it can occur for non-periodic earthquake strong motions of relatively short duration (usually less than about $30 \mathrm{~s}$ of strong shaking) needs to be investigated. This is the subject of this section. 


\subsection{Bending model}

\subsubsection{Finite vertical stiffness}

Clough and Penzien ${ }^{6}$, pp. 522-525 show, from the power spectral density function, that SDOF systems with reasonably low ratios of critical damping $(\xi<0.1)$ can be classified as narrow-band systems. This means that the response of such systems to excitation will locally appear as a slightly distorted sine function with a frequency near the natural frequency of the system with amplitudes that vary slowly in a random fashion. Therefore the response, $u_{t t}^{v}$, of a vertical SDOF system to a strong-motion record can be approximated by $u_{t t}^{v}=A_{v} \cos \left(\omega_{v} t\right)$, where $A_{v}$ is the amplitude and $\omega_{v}$ is the natural angular frequency of the system. Hence Equation 5 becomes:

$$
u_{t t}+2 \xi_{1} \omega_{1} u_{t}+\omega_{1}^{2}\left[1-\beta A_{v} \cos \left(\omega_{v} t\right)\right] u=-U_{t t} .
$$

Therefore parametric resonance is possible if $\beta A_{v}>\beta_{c}$, where $\beta_{c}=A_{V} \beta$ for the critical value of $A_{V} \beta$ for $\omega_{v}$ from Equation 12. Hence if:

$$
A_{v}>\beta_{c} / \beta,
$$

then parametric resonance (leading to large amplification of the horizontal response) can occur if such vertical accelerations are sustained for a long enough time. An upper bound on $A_{v}$ is the maximum spectral acceleration at the period and damping of interest for the vertical strong-motion record; this can be found from acceleration response spectra.

The Tabas N74E and vertical components are used as an example of the importance of parametric resonance with $\xi=0.05$ ( $5 \%$ critical damping in both horizontal and vertical directions) and $\gamma=0.25$. For $\xi=0.05$ have $\beta_{c}=0.2$ (using Figure 11) and for $\gamma=0.25$ have $\beta=0.034$ and therefore for $A_{v}>0.2 / 0.034=5.9 \mathrm{~ms}^{-2}$ parametric resonance is possible

For each of the 46 periods between 0.1 and $2 \mathrm{~s}$ and for $5 \%$ damping the response of the normal SDOF model to the vertical ground motion was calculated and stored. Figure 14 shows the acceleration response spectrum for the vertical component and $5 \%$ damping. Also marked is the period range for which parametric resonance is possible using Inequality 13. This shows that parametric resonance is possible but only for vertical periods shorter than $0.44 \mathrm{~s}$.

The calculated vertical responses are used as the input to calculate the response spectrum, for $5 \%$ damping, of the N74E component using the bending model for $\gamma=0.25$, i.e. solving Equation 5 with $u_{t t}^{v}$ equal to the vertical response accelerations and $-U_{t t}$ equal to the horizontal ground acceleration. Figure 15 shows the percentage increase in spectral acceleration due to the vertical ground motion for 
the response spectrum of the N74E component and the normal SDOF model. As can be seen parametric resonance does occur for this record and it leads to an large increase (over 700\%) in the horizontal spectral acceleration for horizontal and vertical natural periods 0.36 and $0.18 \mathrm{~s}$ respectively.

To assess the importance of parametric resonance generally the same system was subjected to another strong-motion record. To make the comparison valid a search was made of the near-field records to find a vertical time-history with an acceleration spectrum for $5 \%$ damping close to that of the vertical spectrum of the Tabas record (Figure 14). This means that differences in the effect of the vertical excitation are not due simply to the amplitude of the vertical excitation. The vertical time-history which is the closest match, in terms of the acceleration spectrum for $5 \%$ damping, is that from 17645 Saticoy Street from the Northridge earthquake $\left(17 / 1 / 1994, M_{s}=6.8\right)$. Figure 16 shows the acceleration spectrum which can be compared with that of the Tabas record (Figure 14).

The same $\gamma(0.25)$ was used as for the Tabas record and the horizontal response spectrum for each vertical period between 0.1 and $2 \mathrm{~s}$ was computed. Figure 17 shows the percentage increase in spectral acceleration due to the vertical ground motion calculated for the response spectrum of the $180^{\circ}$ component of the 17645 Saticoy Street record and the normal SDOF model.

As can be seen parametric resonance does occur for this record and it leads to an large increase (over $300 \%$ ) in the horizontal spectral acceleration for horizontal and vertical natural periods 0.30 and $0.15 \mathrm{~s}$ respectively. Comparing Figures 15 and 17 shows that although parametric resonance does occur for the 17645 Saticoy Street record, as predicted, it does not greatly increase the response as it does for the Tabas record. This is probably due to the shorter duration of large amplitude motion in the 17645 Saticoy Street record compared with the Tabas record. This difference in duration is shown in Figure 18 where the vertical acceleration time-histories of these two records are compared.

The strong ground motion in the Tabas record lasts longer than that in the 17645 Saticoy Street record because the Tabas earthquake $\left(M_{s}=7.3\right)$ is larger than the Northridge earthquake $\left(M_{s}=6.8\right)$. Therefore because the large amplitude vertical responses required for parametric resonance do not occur for as long in the 17645 Saticoy Street record there is less chance of such resonance causing large increases in the horizontal response compared with the Tabas record. Therefore whether parametric resonance causes large increases in the horizontal response for a particular record is not simply due to the amplitude of the vertical excitation acceleration being large enough so that Inequality 13 holds but also that these large excitations last for a sufficiently long time.

Figures $15 \& 17$ also show that when parametric resonance does not occur the amplifications due to vertical ground motion are small (less than about 10 or $20 \%$ ). Hence if parametric resonance does not occur then vertical ground motion does not have a large effect on horizontal response. 
Figure 19 shows the regions in which parametric resonance can occur in terms of vertical input acceleration, horizontal damping, $\xi$, and load ratio, $\gamma$, using Inequality 13 for the bending model. For example, this graph shows that for a constant harmonic input vertical acceleration with amplitude $5 \mathrm{~ms}^{-2}$ parametric resonance will occur for a horizontal natural period equal to twice the period of the vertical acceleration if the horizontal damping is equal to $5 \%$ and the load ratio is greater than about 0.3 . If the horizontal damping is increased to $10 \%$ then the load ratio needs to be increased to about 0.45 for parametric resonance to occur.

As noted above instability occurs for some of the near-field records for $\gamma>0.1$. This is much lower than load ratios in most structures. Therefore no equations for the prediction of spectral acceleration given magnitude, distance and site category using the bending model and finite vertical stiffness were derived using the near-field dataset.

\subsubsection{Infinite vertical stiffness}

Equation 13 can be used to get a lower limit on the amplitude of the vertical ground acceleration required for parametric resonance. However because ground motions are non-harmonic this is a poor estimator of whether parametric resonance will occur.

Figure 12 shows that parametric resonance can occur for infinite vertical stiffness and bending models. Figure 12(a) clearly shows three peaks of large amplifications (up to about $600 \%$ ) due to the vertical ground acceleration. These peaks occur at natural horizontal periods: $0.12,0.36$ and $0.42 \mathrm{~s}$, which are double the periods at which the largest vertical accelerations occur (see Figure 14) showing that these amplifications are due to parametric resonance. These large amplifications though are not present if the damping is increased to $2 \%$ (see Figure 12(b)) even though Figure 19 shows that parametric resonance is still possible (the graph should be considered for a vertical input acceleration equal to vertical PGA which is $7.3 \mathrm{~ms}^{-2}$ for this record).

Equations for the prediction of spectral acceleration given magnitude, distance and site category using the standard and bending models were derived using the near-field dataset, for details see Ambraseys and Douglas ${ }^{19},{ }^{20}$.

The inclusion of the vertical ground motion has little effect. Figure 13 shows the ratio between the spectral acceleration including the effect of the vertical accelerations and not including the vertical accelerations (note that this ratio is between models not including soil terms). For a site on the surface projection of the rupture plane (i.e. $d=0 \mathrm{~km}$ ) of an earthquake with $M_{s}=7.8$ the increase due to the vertical accelerations is about $8 \%$ and for smaller magnitudes and larger distances it is less. Therefore the effect of vertical excitation on this type of SDOF system can be neglected when it stays stable. 


\subsection{Hinging model}

The situation for the hinging model is more complicated because $\beta$ is dependent on the natural horizontal period of the system and also the length of the column.

\subsubsection{Finite vertical stiffness}

Figure 23 shows the acceleration response spectrum for the vertical component of the Tabas record for $5 \%$ damping. Also marked is the period range for which parametric resonance is possible using Inequality 13 with $l=0.5 \mathrm{~m}$ and horizontal damping of $2 \%$. This shows that for vertical periods longer than $0.65 \mathrm{~s}$ parametric resonance is possible but that for shorter periods than $0.65 \mathrm{~s}$ it is impossible.

The calculated vertical responses are used as the input to calculate the response spectrum, for $2 \%$ damping, of the N74E component using the hinging model for $l=0.5 \mathrm{~m}$, i.e. solving Equation 5 with $u_{t t}^{v}$ equal to the vertical response accelerations and $-U_{t t}$ equal to the horizontal ground acceleration. Figure 24 shows the percentage increase in spectral acceleration due to the vertical ground motion calculated using the response spectrum of the N74E component using the normal SDOF model.

As can be seen parametric resonance does occur for this record and it leads to an large increase (almost 400\%) in the horizontal spectral acceleration for horizontal and vertical natural periods 1.9 and $0.95 \mathrm{~s}$ respectively. Also for short vertical periods (about $0.2 \mathrm{~s}$ ), corresponding to the peak in the vertical response spectrum there is also a large increase in horizontal response for long horizontal periods which is not caused by parametric resonance.

To assess the importance of parametric resonance generally the same system was subjected to another strong-motion record. Figure 25 shows the acceleration spectrum of the 17645 Saticoy Street record and the curve showing the period ranges where parametric resonance is possible for $l=0.5 \mathrm{~m}$, vertical damping $5 \%$ and horizontal damping $2 \%$, which can be compared with that of the Tabas record (Figure 23).

The same $l(0.5 \mathrm{~m})$ was used as for the Tabas record and the horizontal response spectrum for each vertical period between 0.1 and $2 \mathrm{~s}$ was computed for $2 \%$ damping. Figure 26 shows the percentage increase in spectral acceleration due to the vertical ground motion calculated using the response spectrum of the $180^{\circ}$ component of the 17645 Saticoy Street record using the normal SDOF model.

As can be seen parametric resonance does occur for this record and it leads to an large increase (almost $300 \%$ ) in the horizontal spectral acceleration for horizontal and vertical natural periods 1.70 and 0.85 s respectively. Comparing Figures 24 and 26 shows that although parametric resonance does occur for the 17645 Saticoy Street record, as is predicted, it does not greatly increase the response as does the Tabas record. This is probably due to the smaller duration of large amplitude motion in the 17645 
Saticoy Street record compared with the Tabas record. The 17645 Saticoy Street record increases the horizontal response for short vertical periods and long horizontal periods (Figure 26) in the same way as the Tabas record (Figure 24). Note however that this choice of length of column, $l=0.5 \mathrm{~m}$, is unrealistic for normal structures.

Figure 27 shows the regions in which parametric resonance can occur in terms of vertical input acceleration, horizontal damping, $\xi$, natural horizontal period and length of column, $l$, using Inequality 13 for the hinging model. For example, this graph shows that for a constant harmonic input vertical acceleration with amplitude $5 \mathrm{~ms}^{-2}$ parametric resonance will occur for a horizontal natural period equal to twice the period of the vertical acceleration if the horizontal damping is equal to $5 \%$, the length of the column is equal to $1 \mathrm{~m}$ and the natural horizontal period is greater than about $1.3 \mathrm{~s}$. If the length of the column is increased to $5 \mathrm{~m}$ then the horizontal damping needs to be decreased to about $1 \%$ for parametric resonance to occur.

Most vertical strong-motion records, even in the near field, do not contain enough energy in the long period range for parametric resonance (defined by the regions of Figure 27) to occur. Figure 27 shows that parametric resonance is most likely for long vertical periods $(T>1 \mathrm{~s})$, very few structures though have such a vertical period and hence it is unlikely that parametric resonance will lead to large increases in the horizontal response of structures that can be modelled by SDOF systems with hinging.

Equations for the prediction of spectral acceleration given magnitude, distance and site category have been derived for the hinging model with finite vertical stiffness, for $5 \%$ horizontal and vertical damping, $l=2 \mathrm{~m}$ and 46 horizontal and vertical periods between 0.1 and $2 \mathrm{~s}$, for details see Ambraseys and Douglas ${ }^{19}$. Figure 28 shows a contour plot of the ratio between the predicted spectral acceleration when vertical ground motion is included (finite vertical stiffness hinging model for $l=2 \mathrm{~m}$ ) and the predicted spectral acceleration when it is ignored for 7.8 at distance $0 \mathrm{~km}$. The maximum increase due to the vertical excitation is about $25 \%$ which occurs for a horizontal natural period of about $2 \mathrm{~s}$ and a vertical natural period of about $1 \mathrm{~s}$ (Figure 28) and so is probably due to parametric resonance which occurs for vertical periods which are half the horizontal period. The effect of vertical excitation on this type of SDOF system can be neglected even when the vertical stiffness is finite.

\subsubsection{Infinite vertical stiffness}

Hjelmstad and Williamson ${ }^{22}$ state for the hinging model '[i]t is evident from the preceding discussion [about parametric resonance leading to unbounded responses] that parametric resonance associated with vertical motions, could be a concern in earthquake response of structures if the input motion exhibits near periodicity, as was true in the 1985 earthquake, experienced in the the Mexico City lake bed region. One 
should note that the values of $\eta$ [here called $\beta$ ] in building structures are typically rather small, thereby limiting the troublesome range of frequencies associated with parametric resonance.'

This idea was tested using a record of the Michoacán (19/9/1985) from Mexico City (CDAF de Abastos Oficia) which is on very soft soil $\left(V_{s, 30}=61 \mathrm{~ms}^{-1}\right)$ and exhibits sinusoidal (see Figure 20) ground motion with period about 2 to $3 \mathrm{~s}$. Figure 21 shows the percentage increase in the horizontal spectral acceleration due to the vertical ground motion for this record with $l=0.25 \mathrm{~m}, 5 \%$ vertical damping and $0 \%$ horizontal damping. From Figure 21 it can be seen that there is an increase in the horizontal response due to parametric resonance at periods greater than about $3 \mathrm{~s}$. However, the length of column required to cause this increase is not realistic.

Equations for the prediction of spectral acceleration given magnitude, distance and site category using the standard and hinging models were derived using the near-field dataset, for details see Ambraseys and Douglas ${ }^{19},{ }^{20}$.

The inclusion of the vertical ground motion has little effect. Figure 22 shows the ratio between the spectral acceleration with and without the effect of the vertical accelerations (note that this ratio is between models not including soil terms). For a site $0 \mathrm{~km}$ from an earthquake with $M_{s}=7.8$ the increase due to the vertical accelerations is about $9 \%$ and for smaller magnitudes and larger distances it is less. Therefore the effect of vertical excitation on this type of SDOF system can be neglected when it stays stable.

\section{Conclusions}

The two elastic SDOF models studied for this article, the bending and the hinging models, both have three main types of behaviour: normal, parametric resonance and instability. The type of behaviour the system exhibits is controlled by the combination of system parameters and the vertical input acceleration.

The systems are unstable when the multiplier of horizontal displacement in the equation of the motion is negative for a sufficiently long period of time so that exponential solutions of the equation are possible and the systems collapse because the displacement (and velocity and accelerations) tend to infinity. This limit is simply the stability criterion that the system must obey in the static case modified due to vertical ground motion.

The length of interval above the critical acceleration required to induce instability is related to the horizontal natural period of the system: short period systems require shorter intervals than long period systems. The length of interval of above critical accelerations required for instability in bending systems with periods between 0.1 and $2 \mathrm{~s}$ is about $0.05 \mathrm{~s}$. The length of interval of above critical accelerations required for instability in hinging systems with periods between 0.1 and $2 \mathrm{~s}$ is greater than that for the 
bending model and is equal to about $0.2 \mathrm{~s}$. Size of horizontal damping of either system has little effect on the length of time that is required to cause instability.

A number of vertical records do induce instability in SDOF models with bending and finite vertical stiffness for load ratios of about 0.3 to 0.5 . Therefore such a failure mechanism is possible for structures that can be modelled by such SDOF models.

No recorded vertical ground motions induce instability in SDOF systems with hinging for realistic length of columns (greater than $1 \mathrm{~m}$ ) and horizontal and vertical damping and period. Therefore such a failure mechanism is not possible for structures that can be modelled by such SDOF models.

The systems exhibit parametric resonance when the amplitude of the vertical acceleration is greater than a limit acceleration and the period of this vertical acceleration is half the natural horizontal period. This limit acceleration depends on the structural parameters: horizontal damping and length of column (for the hinging model) or load ratio (for the bending model). Parametric resonance can lead to large increases (up to $700 \%$ ) in the horizontal response of bending systems with realistic structural parameters. Although parametric resonance can lead to large increases (up to 300\%) in the horizontal response of hinging systems these increases are for unrealistic structural parameters, i.e. extremely short columns with large horizontal and vertical periods so parametric resonance is not likely to occur in structures that approximate to hinging models. The duration of the strong motion affects the size of the increase in horizontal response due to parametric resonance so longer durations of strong motion lead to large increases in response because parametric resonance can build up. For infinite vertical stiffness parametric resonance can occur but this is only for structural parameters which are unlikely to occur in practice.

When the combination of system parameters and vertical input accelerations means that instability and parametric resonance do not occur then the system behaves almost the same as the ordinary zerogravity system defined by Equation 1. The amplifications due to the vertical excitation are small. For most vertical ground motions and realistic choices of system parameters this is the type of behaviour which will occur.

\section{Acknowledgements}

This study was funded by EPSRC grant number GR/L87385; we thank them for their support. Also, we thank Drs. K. Simpson, M. Srbulov, M. Free and S.K. Sarma and P. Smit for their help in this research. We thank three anonymous reviewers for their comments, which significantly improved this paper. 


\section{References}

[1] Chopra AK. Dynamics of Structures - Theory and Application to Earthquake Engineering. Prentice Hall International, Inc., 1995.

[2] Lin YK, Shih TY. Column response to horizontal-vertical earthquakes. Journal of The Engineering Mechanics Division, ASCE 1980; 106(EM6): 1099-1109.

[3] Orabi II, Ahmadi G. Response of structures subjected to horizontal-vertical random earthquake excitations. Soil Dynamics and Earthquake Engineering 1988; 7(1): 9-14.

[4] Loh CH, Ma MJ. Reliability assessment of structures subjected to horizontal-vertical random earthquake excitations. Structural Safety 1997; 19(1): 153-168.

[5] Şafak E. A simple method to account for the effects of vertical loads on the horizontal seismic response of buildings. Proceedings of the Sixth International Conference on Seismic Zonation. 2000 .

[6] Clough RW, Penzien J. Dynamics of Structures. 2nd edition. McGraw-Hill, Inc., New York, USA, and London, UK, 1993.

[7] Bycroft GN. White noise representation of earthquake. Journal of The Engineering Mechanics Division, ASCE 1960; 86(EM2): 1-16.

[8] Liu SC, Jhaveri DP. Spectral and correlation analysis of ground-motion accelerograms. Bulletin of the Seismological Society of America 1969; 59(4): 1517-1534.

[9] Lin YK. Email message received 23/5/2000, 2000.

[10] Shih TY, Lin YK. Vertical seismic load effect on hysteric columns. Journal of The Engineering Mechanics Division, ASCE 1982; 108(EM2): 242-254.

[11] Jennings PC, Husid R. Collapse of yielding structures during earthquakes. Journal of The Engineering Mechanics Division, ASCE 1968; 94(EM5): 1045-1065.

[12] Husid R. The effect of gravity on the collapse of yielding structures with earthquake excitation. Proceedings of Fourth World Conference of Earthquake Engineering, volume II. 1969 A-4 31-43.

[13] Sun CK, Berg GV, Hanson RD. Gravity effect on single-degree inelastic system. Journal of The Engineering Mechanics Division, ASCE 1973; 99(EM1): 183-200.

[14] Bernal D. Amplification factors for inelastic dynamic P- $\Delta$ effects in earthquake analysis. Earthquake Engineering and Structural Dynamics 1987; 15(5): 635-651.

[15] Fenwick RC, Davidson BJ, Chung BT. P-Delta actions in seismic resistant structures. Bulletin of the New Zealand National Society for Earthquake Engineering 1992; 25(1): 56-69.

[16] Tani S, Soda S. Vertical load effect on structural dynamics. Proceedings of Sixth World Conference on Earthquake Engineering, volume II. 1977 1028-1033. 
[17] Newmark NM, Rosenblueth E. Fundamentals of Earthquake Engineering. Prentice-Hall, Inc., Englewood Cliffs, New Jersey, USA, 1971.

[18] Newmark NM, Hall WJ. Earthquake Spectra and Design. Earthquake Engineering Research Institute, 1987.

[19] Ambraseys N, Douglas J. Reappraisal of the effect of vertical ground motions on response. ESEE Report 00-4, Department of Civil and Environmental Engineering, Imperial College, London, 2000.

[20] Ambraseys NN, Douglas J. Near-field horizontal and vertical earthquake ground motions. Soil Dynamics and Earthquake Engineering 2003; 23(1): 1-18.

[21] Bolotin VV. The Dynamic Stability of Elastic Systems. Holden-Day, Inc., 1964. Translated by V.I. Weingarten, L.B. Greszczuk, K.N. Trirogoff and K.D. Gallegos.

[22] Hjelmstad KD, Williamson EB. Dynamic stability of structural systems subjected to base excitation. Engineering Structures 1998; 20(4-6): 425-432. 


\begin{tabular}{l|l|l}
$\begin{array}{l}\text { Damping } \\
\text { ratio (\%) }\end{array}$ & $\begin{array}{l}\text { Min. and max. } \\
\text { interval lengths }(\mathrm{s})\end{array}$ & $\begin{array}{l}\text { Most common } \\
\text { interval lengths }(\mathrm{s})\end{array}$ \\
\hline 0 & 0 and 0.05 & 0 to 0.03 \\
2 & 0 and 0.06 & 0 to 0.04 \\
5 & 0.005 and 0.08 & 0.02 to 0.06 \\
10 & 0.01 and 0.08 & 0.02 to 0.07 \\
20 & 0.01 and 0.13 & 0.04 to 0.09
\end{tabular}

Table 1: Minimum, maximum and the most common length of intervals, for which the vertical acceleration is above the limit that causes instability in bending model.

\begin{tabular}{ll|ll}
$T_{h}$ & $\begin{array}{l}\text { Length of } \\
(\mathrm{s})\end{array}$ & $\begin{array}{l}T_{h} \\
\text { interval }(\mathrm{s})\end{array}$ & $\begin{array}{l}\text { Length of } \\
\text { interval }(\mathrm{s})\end{array}$ \\
\hline 0.01 & 0.01 & 1.0 & 0.11 \\
0.02 & 0.02 & 2.0 & 0.22 \\
0.05 & 0.025 & 5.0 & 0.48 \\
0.1 & 0.03 & 10.0 & 0.50 \\
0.2 & 0.05 & 20.0 & 0.50 \\
0.5 & 0.07 & 50.0 & 0.50
\end{tabular}

Table 2: Horizontal natural period of system against length of interval over the critical acceleration defined by Inequality 10 required to cause instability for the Tabas N74E component and $5 \%$ damping.

\begin{tabular}{l|l|l}
$\begin{array}{l}\text { Damping } \\
\text { ratio (\%) }\end{array}$ & $\begin{array}{l}\text { Min. and max. } \\
\text { interval lengths ( s) }\end{array}$ & $\begin{array}{l}\text { Most common } \\
\text { interval lengths (s) }\end{array}$ \\
\hline 0 & 0.05 to 0.9 & 0.1 to 0.2 \\
2 & 0.05 to 1.0 & 0.1 to 0.25 \\
5 & 0.1 and 1.2 & 0.15 to 0.25 \\
10 & 0.1 and 1.2 & 0.15 to 0.3 \\
20 & 0.1 and 1.3 & 0.15 to 0.3
\end{tabular}

Table 3: Minimum, maximum and the most common length of intervals, for which the vertical acceleration is above limit which causes instability in hinging model. 
Figure captions

1. Structural model for zero gravity field where vertical acceleration is neglected.

2. Bending structural model:

(a) for non-zero gravity field where vertical ground motion is neglected.

(b) for non-zero gravity field where vertical ground motion is considered and vertical stiffness is infinite.

(c) for non-zero gravity field where vertical ground motion is considered and vertical stiffness is finite.

3. Hinging structural model:

(a) for non-zero gravity field where vertical ground motion is neglected.

(b) for non-zero gravity field where vertical ground motion is considered and vertical stiffness is infinite.

(c) for non-zero gravity field where vertical ground motion is considered and vertical stiffness is finite.

4. Factor, $1 / \sqrt{1+T_{1}^{2} g /\left[(2 \pi)^{2} l\right]}$, against $T_{1}$ for length of pendulum, $l=5,10,15,20$ and $25 \mathrm{~m}$.

5. Amplification in maximum spectral acceleration due to vertical excitation against $\gamma$ for Tabas N74E component (from Tabas earthquake, 16/9/1978) (bending model).

6. Amplification in maximum spectral acceleration due to vertical excitation against maximum interval above limit acceleration for Tabas N74E component (bending model).

7. Maximum load ratio against cumulative number of records for which Inequality 10 holds for natural vertical periods between 0.1 and $2 \mathrm{~s}$.

8. Amplification in maximum spectral acceleration due to vertical excitation against $l$ for Tabas N74E component (hinging model).

9. Amplification in maximum spectral acceleration due to vertical excitation against maximum interval above limit acceleration for Tabas N74E component (hinging model).

10. Minimum length of column against cumulative total number of records for which Inequality 11 holds for natural vertical periods between 0.1 and $2 \mathrm{~s}$.

11. Graph showing regions of instability where parametric resonance occurs. Parametric resonance occurs within the region to the right of each line.

12. Percentage increase in spectral acceleration due to the vertical ground motion for infinite vertical stiffness for the N74E component of the Tabas strong-motion record.

(a) Undamped

(b) $2 \%$ damping

13. Ratio between the predicted spectral acceleration for the bending model $(\gamma=0.3)$ and for the standard model.

14. Absolute acceleration response spectrum of the vertical component of the Tabas record for $5 \%$ damping. Dashed line indicates lowest amplitude of vertical acceleration required for parametric resonance for $\gamma=0.25$. 
15. Percentage increase in spectral acceleration due to the vertical ground motion for finite vertical stiffness, $5 \%$ damping and $\gamma=0.25$ for the N74E component of the Tabas strong-motion record.

16. Like Figure 14 but for the vertical component of the 17645 Saticoy Street record from the Northridge (17/1/1994) earthquake.

17. Like Figure 15 but for the $180^{\circ}$ component of the 17645 Saticoy Street strong-motion record.

18. Vertical acceleration time-histories.

(a) 17645 Saticoy Street $\left(M_{w}=6.7, M_{s}=6.8\right)$

(b) Tabas $\left(M_{w}=7.4, M_{s}=7.3\right)$

19. Parametric resonance can occur for combinations of $\xi$ and $\gamma$ which are above line corresponding to the vertical input acceleration.

20. Vertical acceleration time-history from Mexico City (CDAF de Abastos Oficia) of the Michoacán earthquake (19/9/1985).

21. Percentage increase in spectral acceleration due to the vertical ground motion for infinite vertical stiffness, $5 \%$ vertical damping, $0 \%$ horizontal damping and $l=0.25 \mathrm{~m}$ for the N000 component of the Mexico City (CDAF de Abastos Oficia) strong-motion record.

22. Ratio between the predicted spectral acceleration for hinging model $(l=2 \mathrm{~m})$ and for the standard model.

23. Absolute acceleration response spectrum of the vertical component of the Tabas record for $5 \%$ damping. Dashed line marks the lowest amplitude of vertical acceleration required for parametric resonance for $2 \%$ horizontal damping and $l=0.5 \mathrm{~m}$.

24. Percentage increase in spectral acceleration due to the vertical ground motion for finite vertical stiffness, $5 \%$ damping vertically and $2 \%$ damping horizontally and $l=0.5 \mathrm{~m}$ for the N74E component of the Tabas strong-motion record.

25. Like Figure 23 but for the vertical component of the 17645 Saticoy Street record.

26. Like Figure 24 but for the $180^{\circ}$ component of the 17645 Saticoy Street strong-motion record.

27. Parametric resonance can occur for combinations of $\xi, T$ and $l$ which are above line corresponding to the vertical input acceleration.

(a) $l=1 \mathrm{~m}$

(b) $l=5 \mathrm{~m}$

28. Ratio between the predicted spectral acceleration for hinging model $(l=2 \mathrm{~m})$ and the standard model $\left(M_{s}=7.8, d=0 \mathrm{~km}\right)$. 


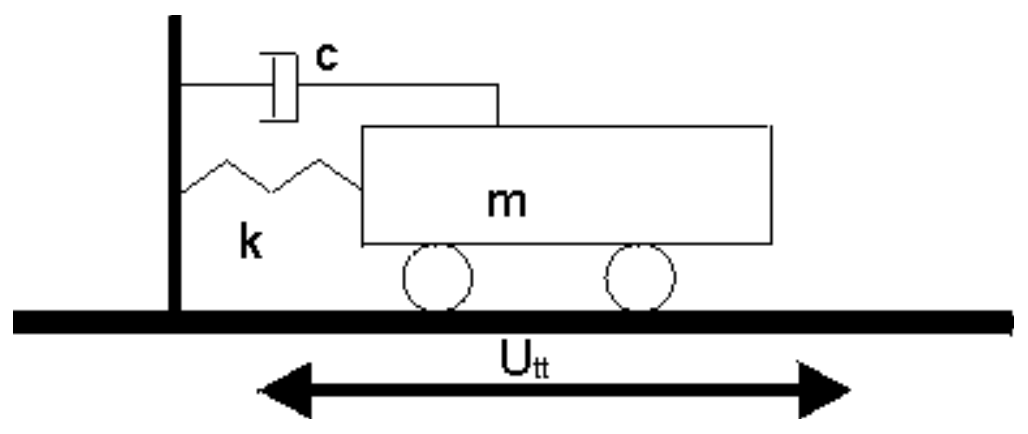

Figure 1:

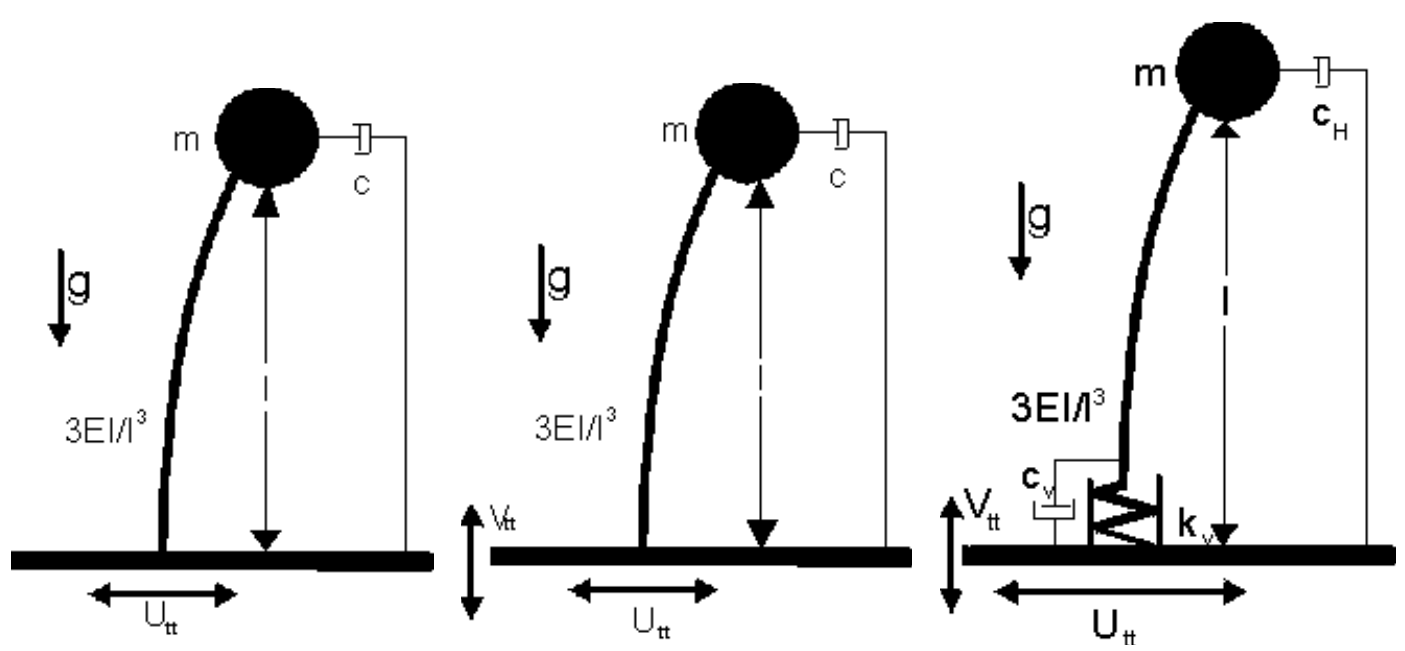

(a)

(b)

(c)

Figure 2:

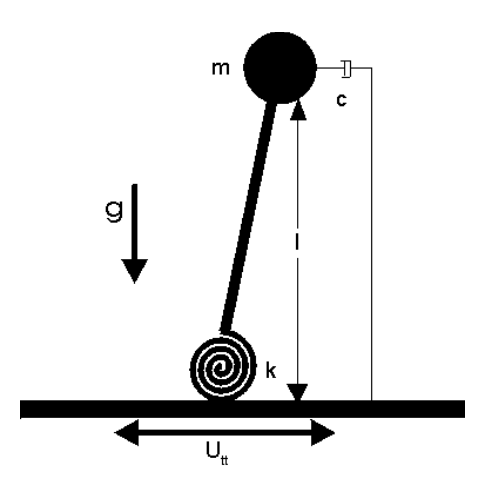

(a)

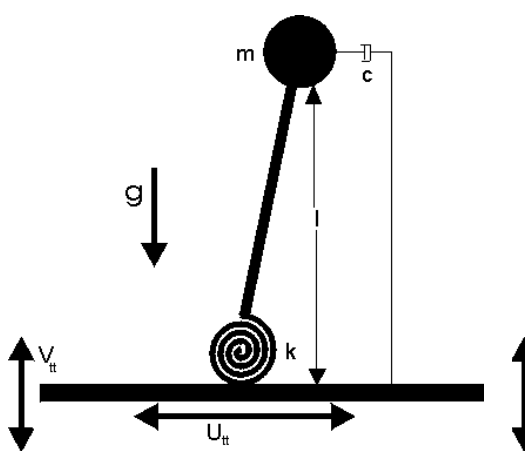

(b)

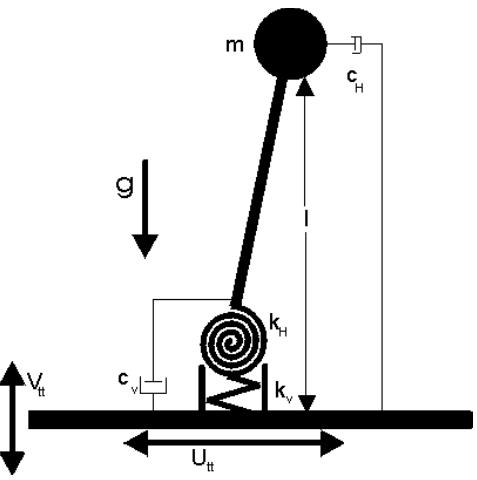

(c)

Figure 3: 


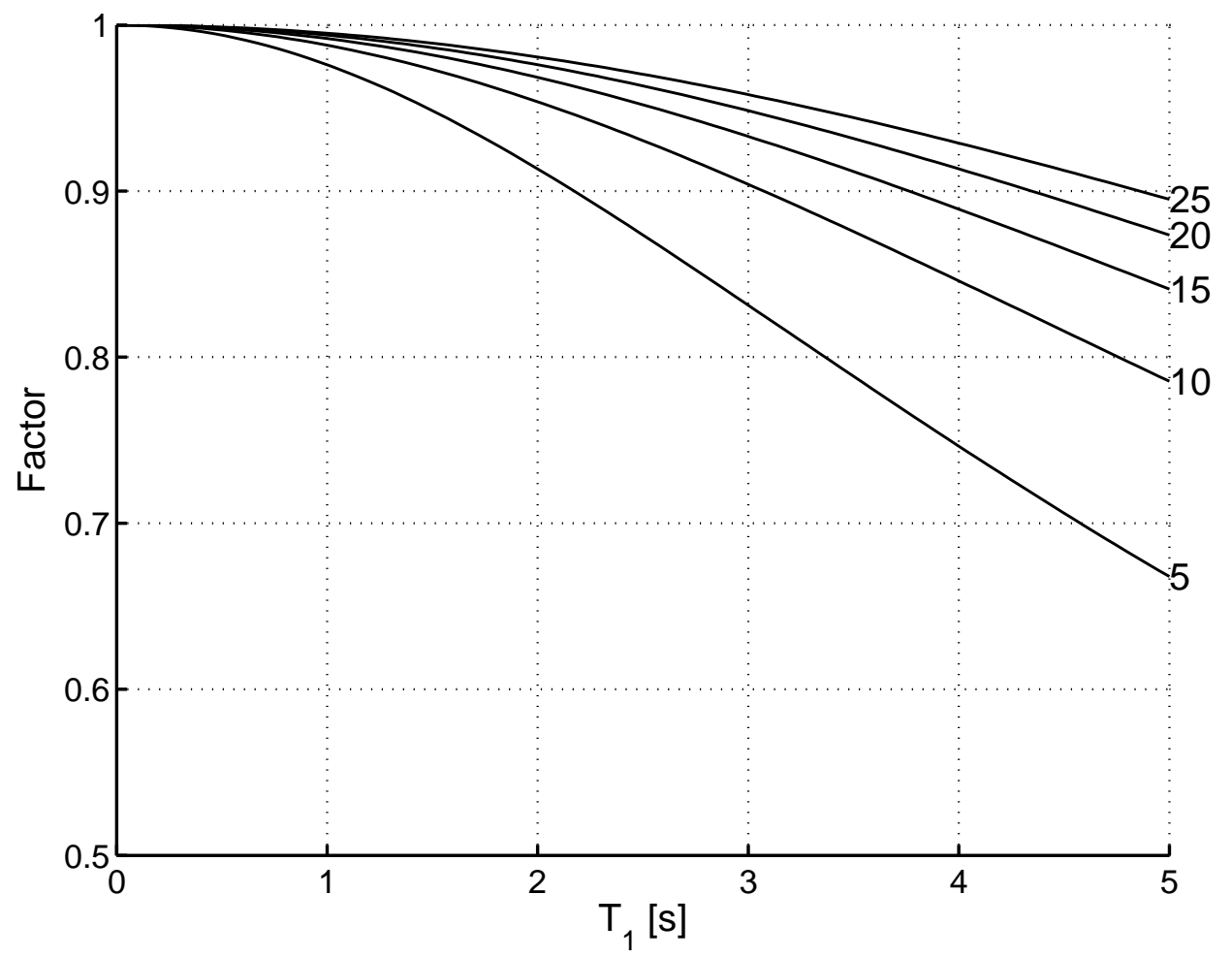

Figure 4:

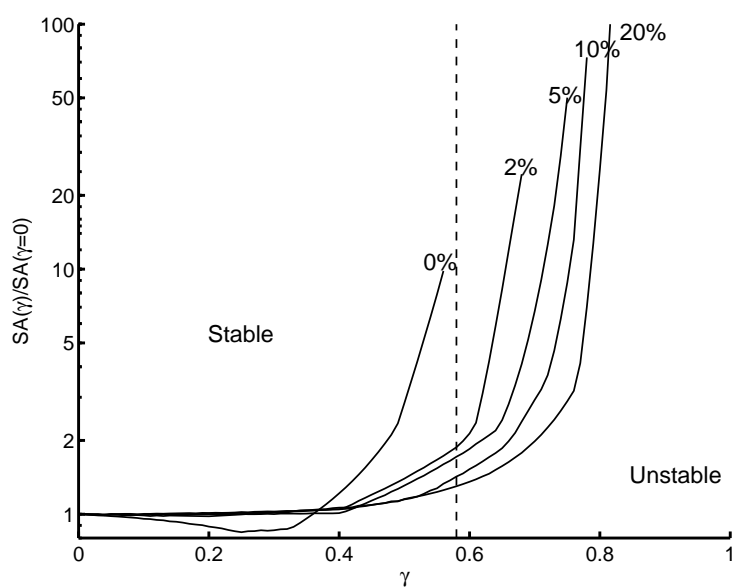

Figure 5:

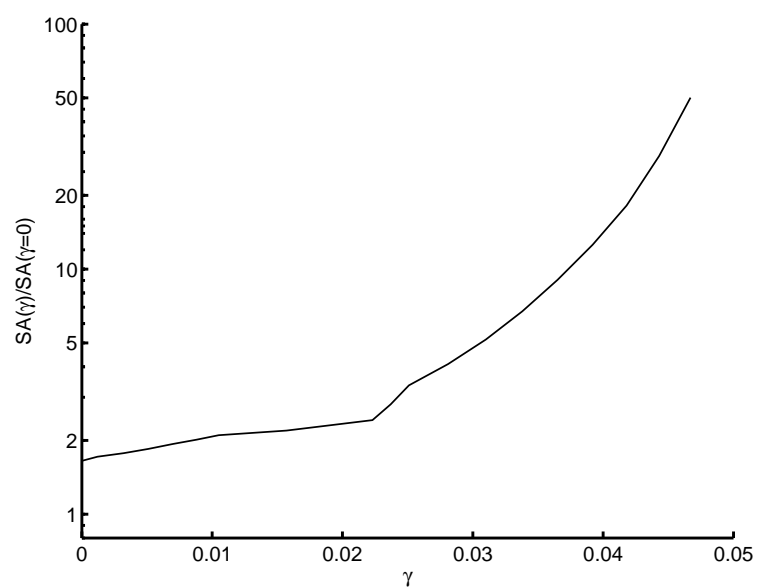

Figure 6: 


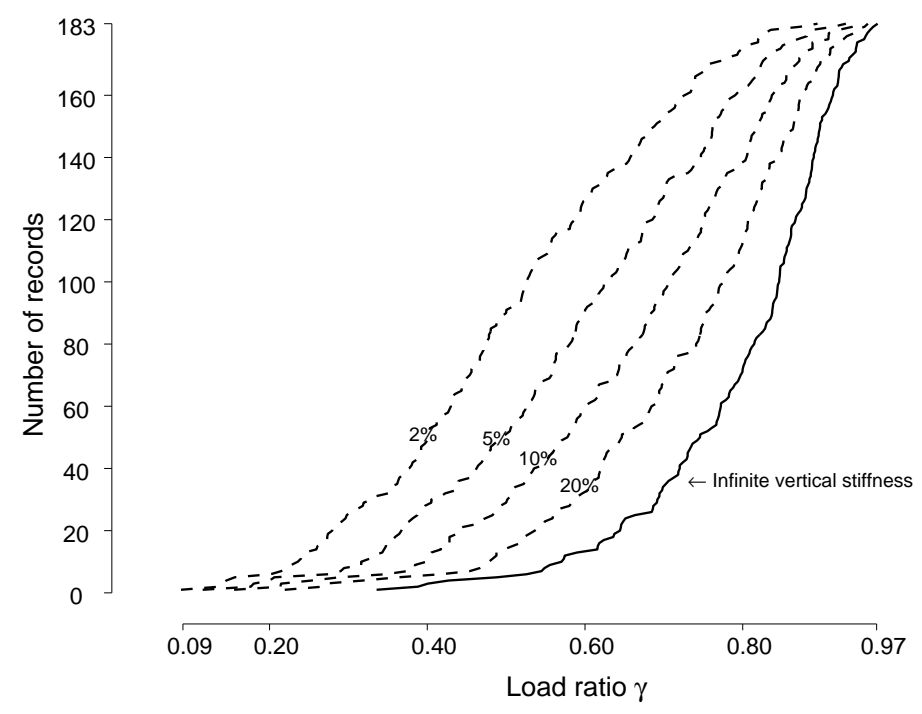

Figure 7:
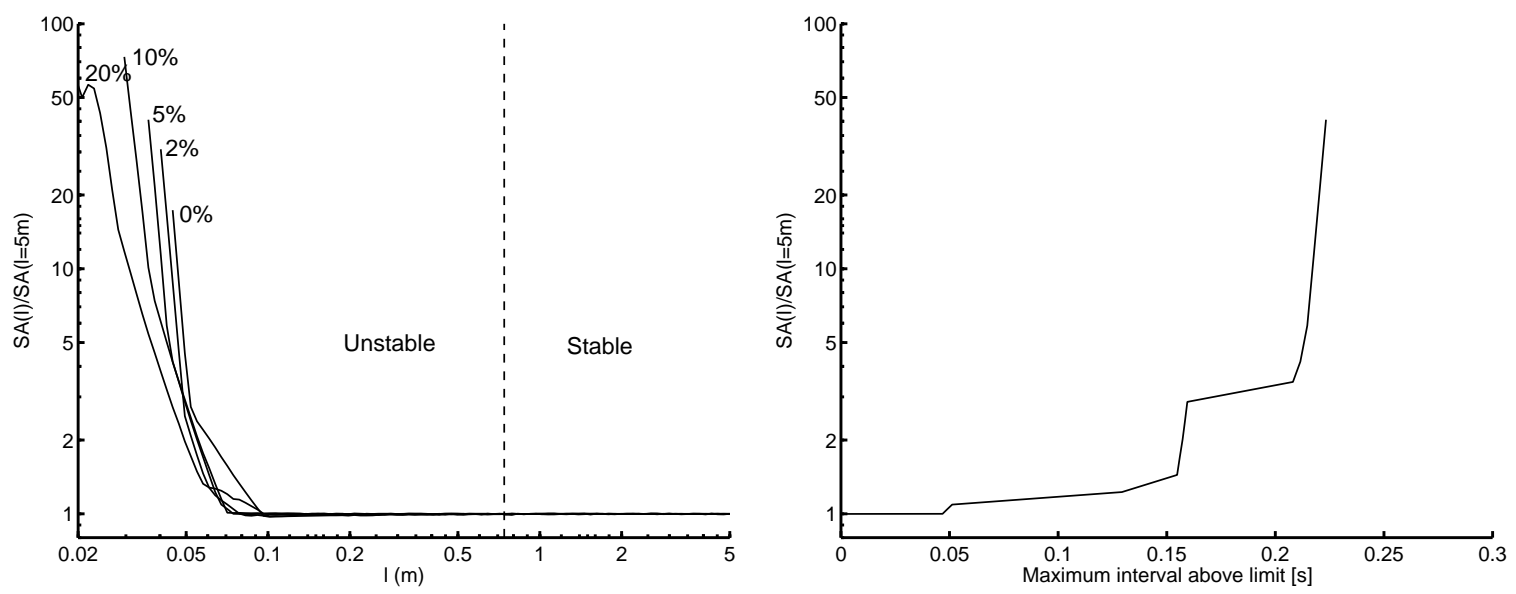

Figure 8:

Figure 9: 


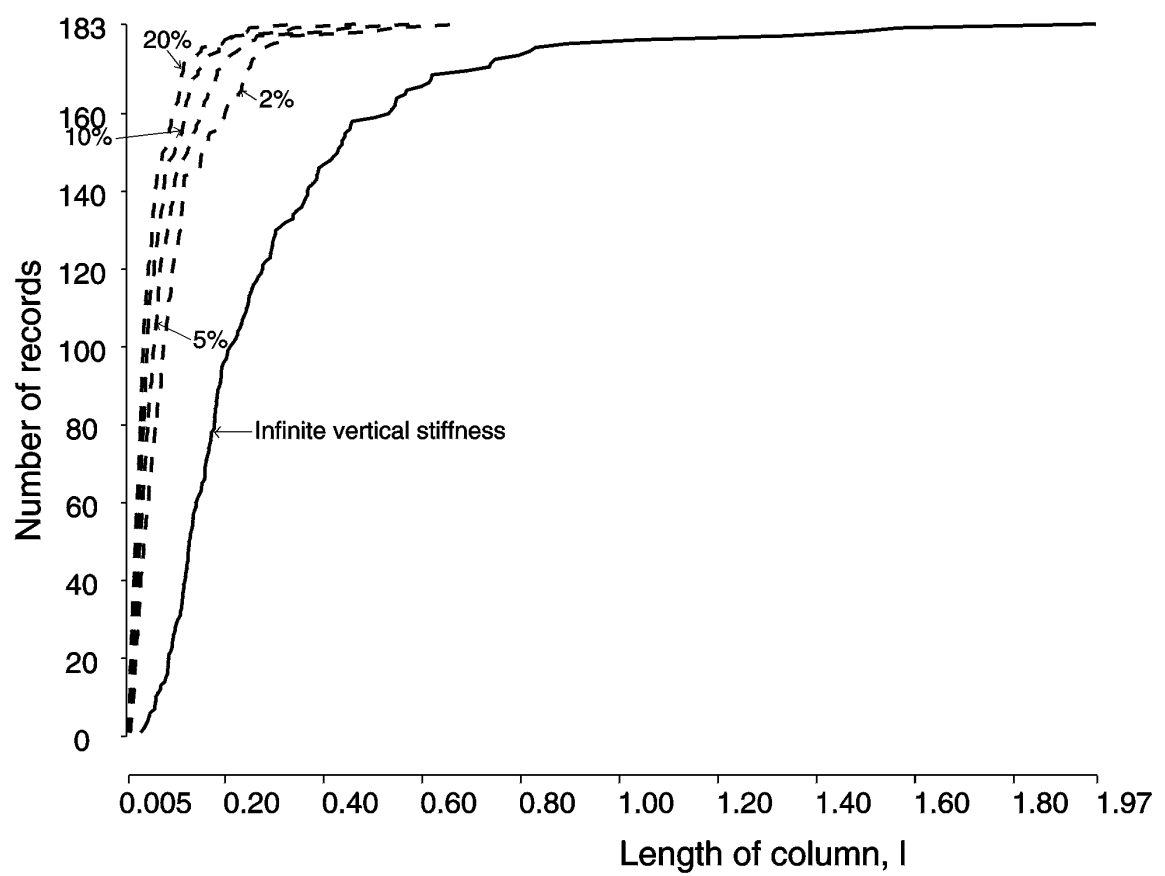

Figure 10:

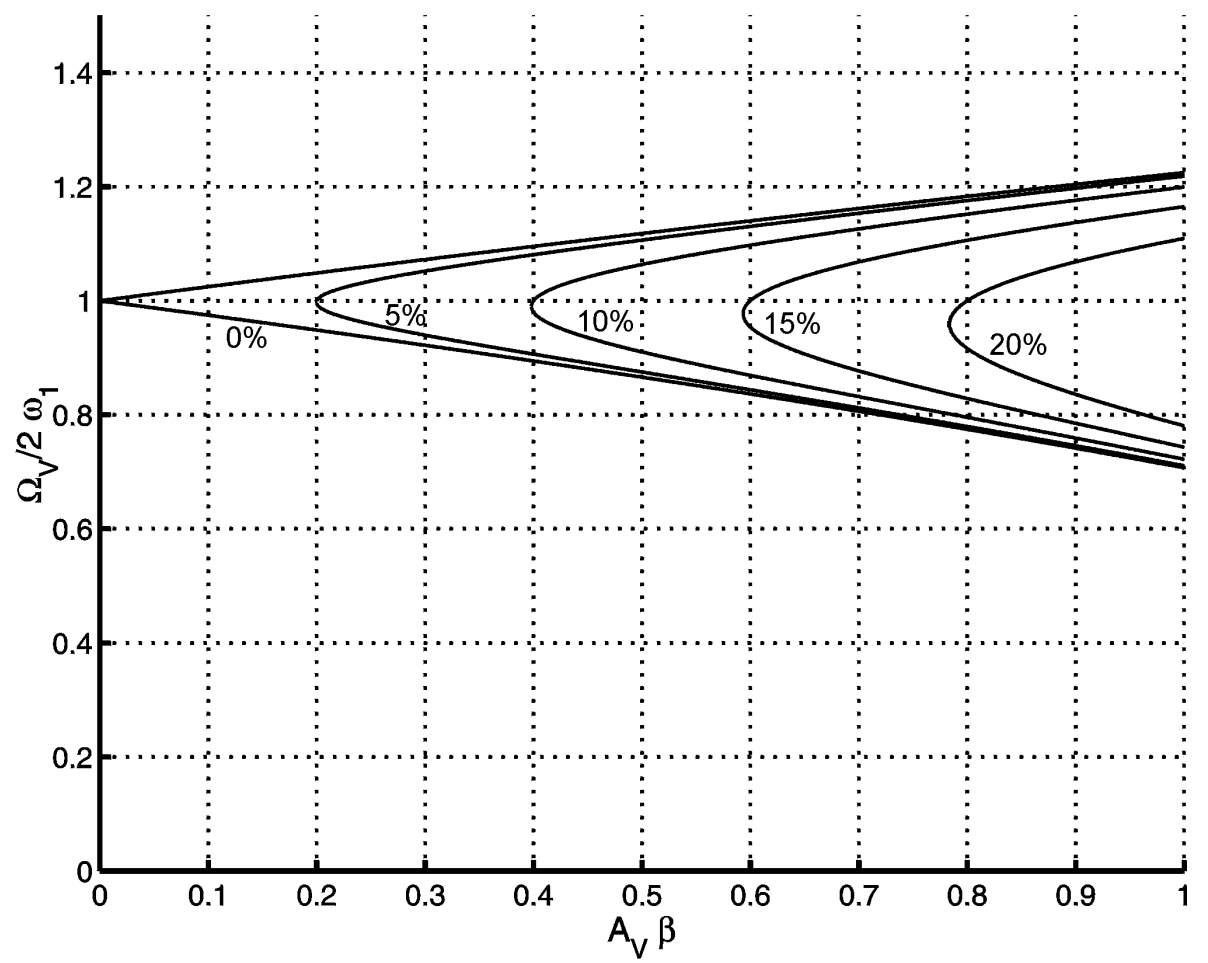

Figure 11: 


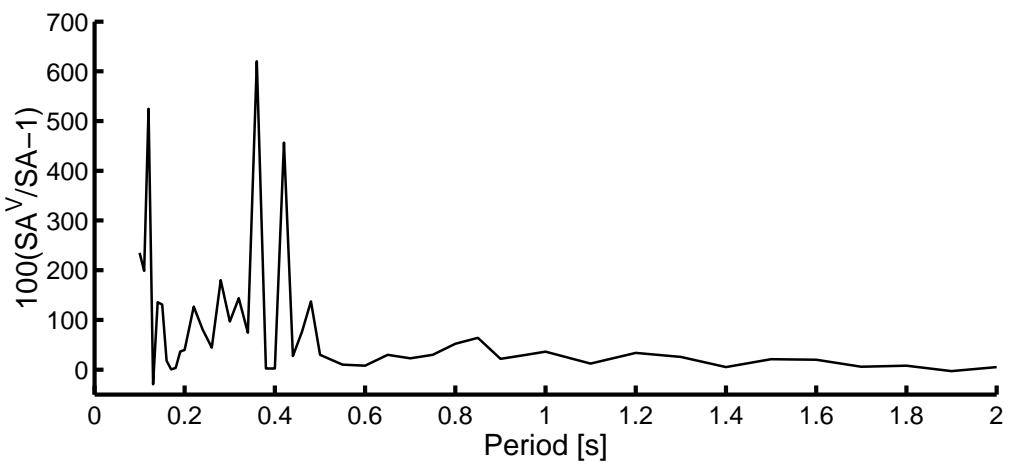

(a)

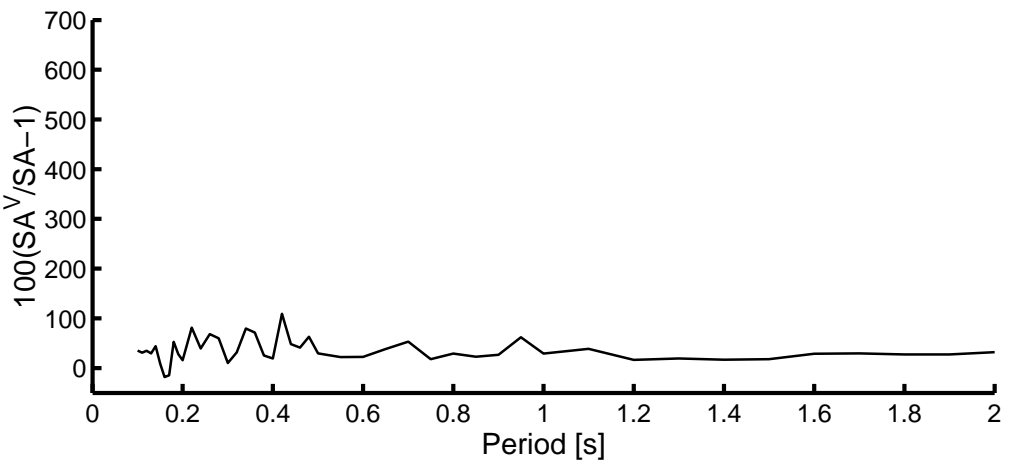

(b)

Figure 12: 


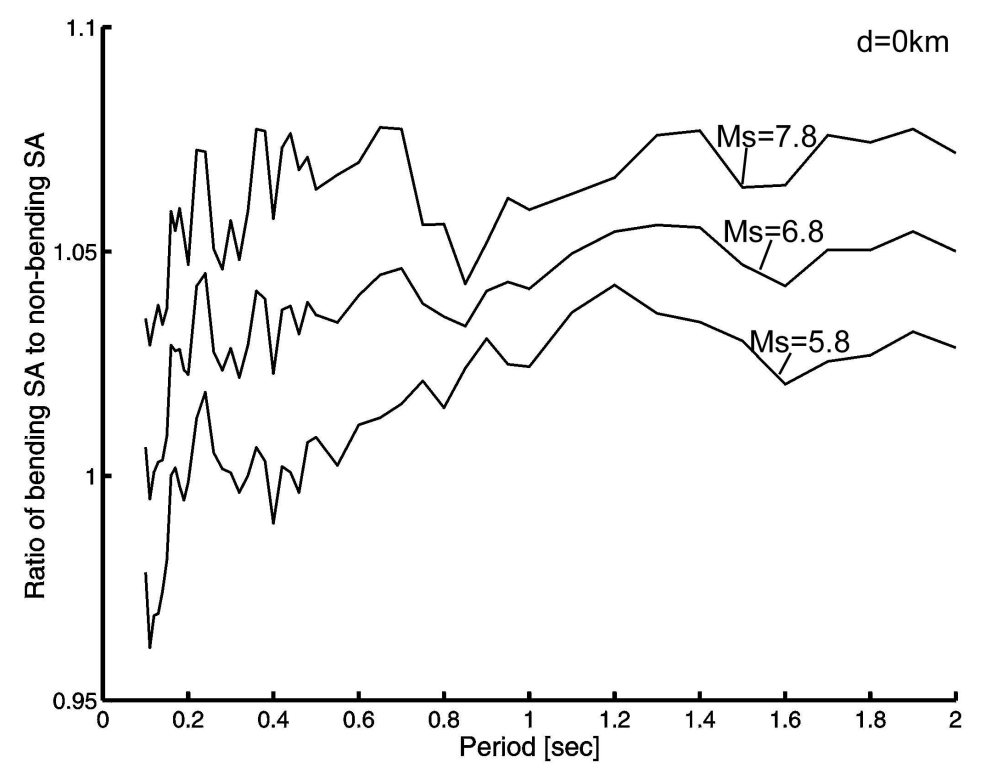

Figure 13:

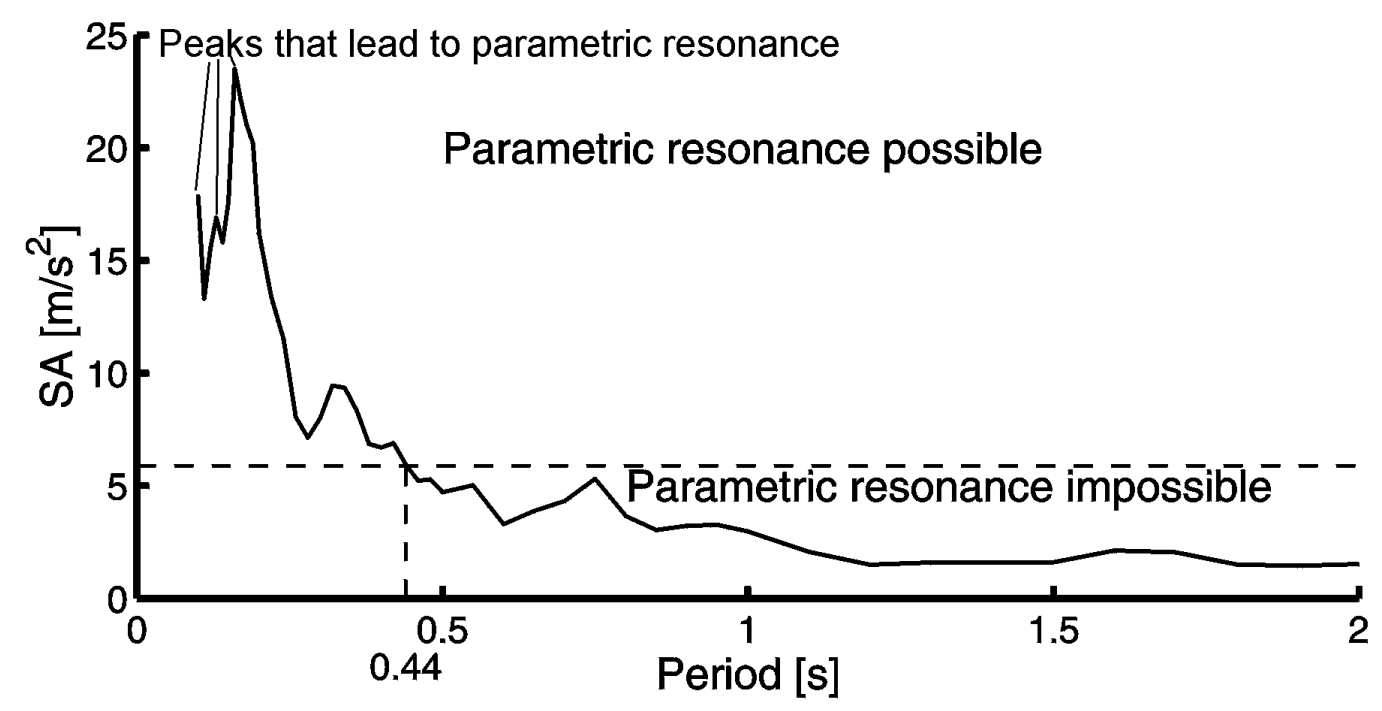

Figure 14: 


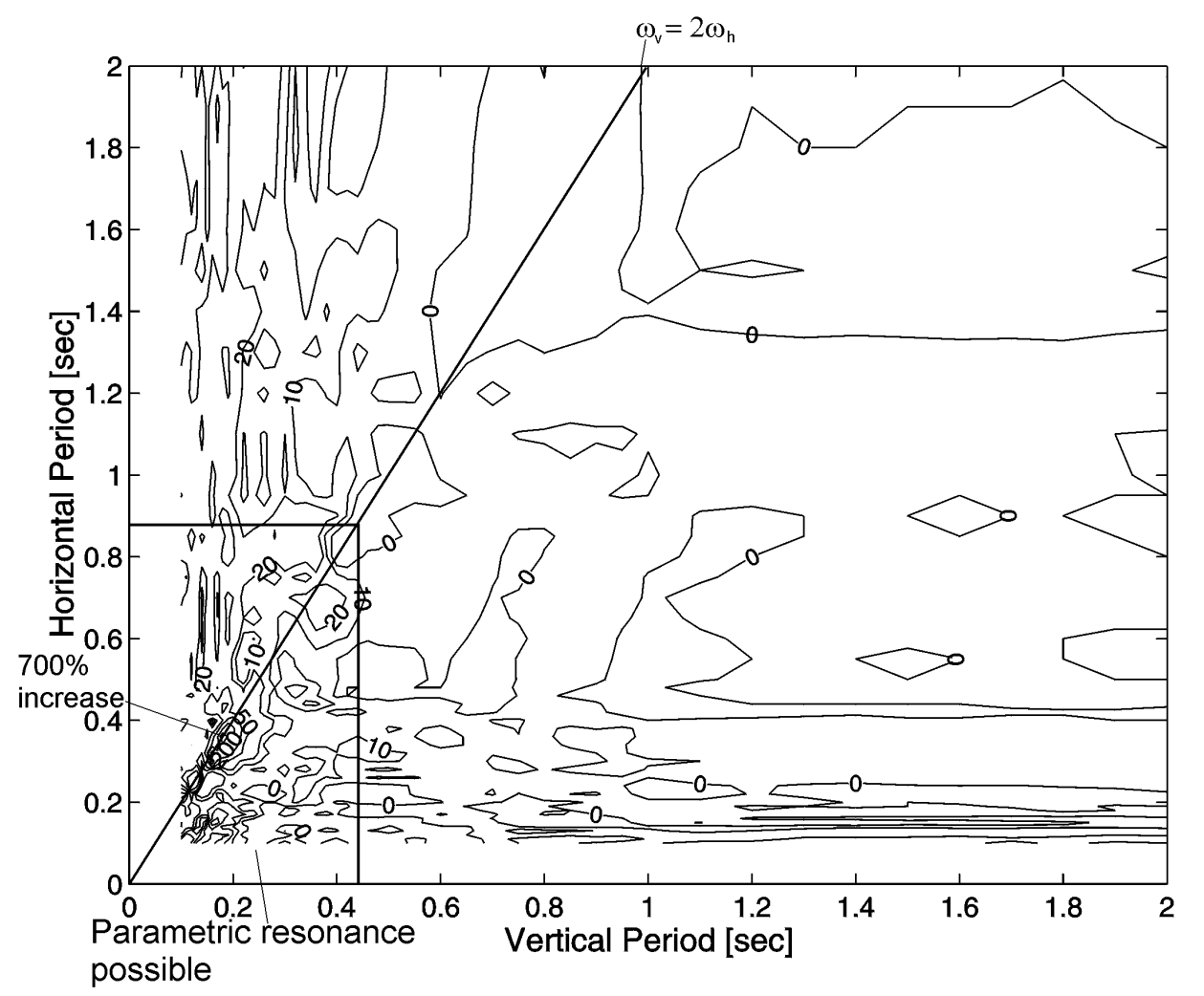

Figure 15: 


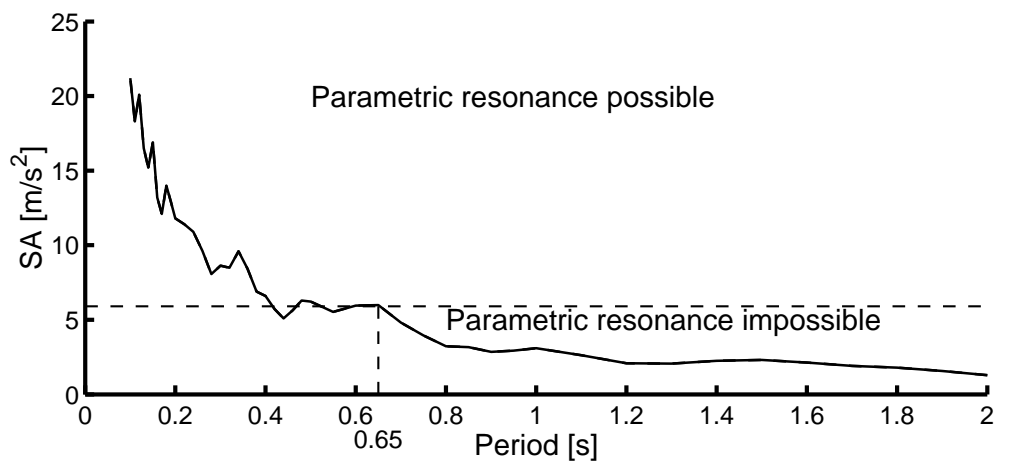

Figure 16:

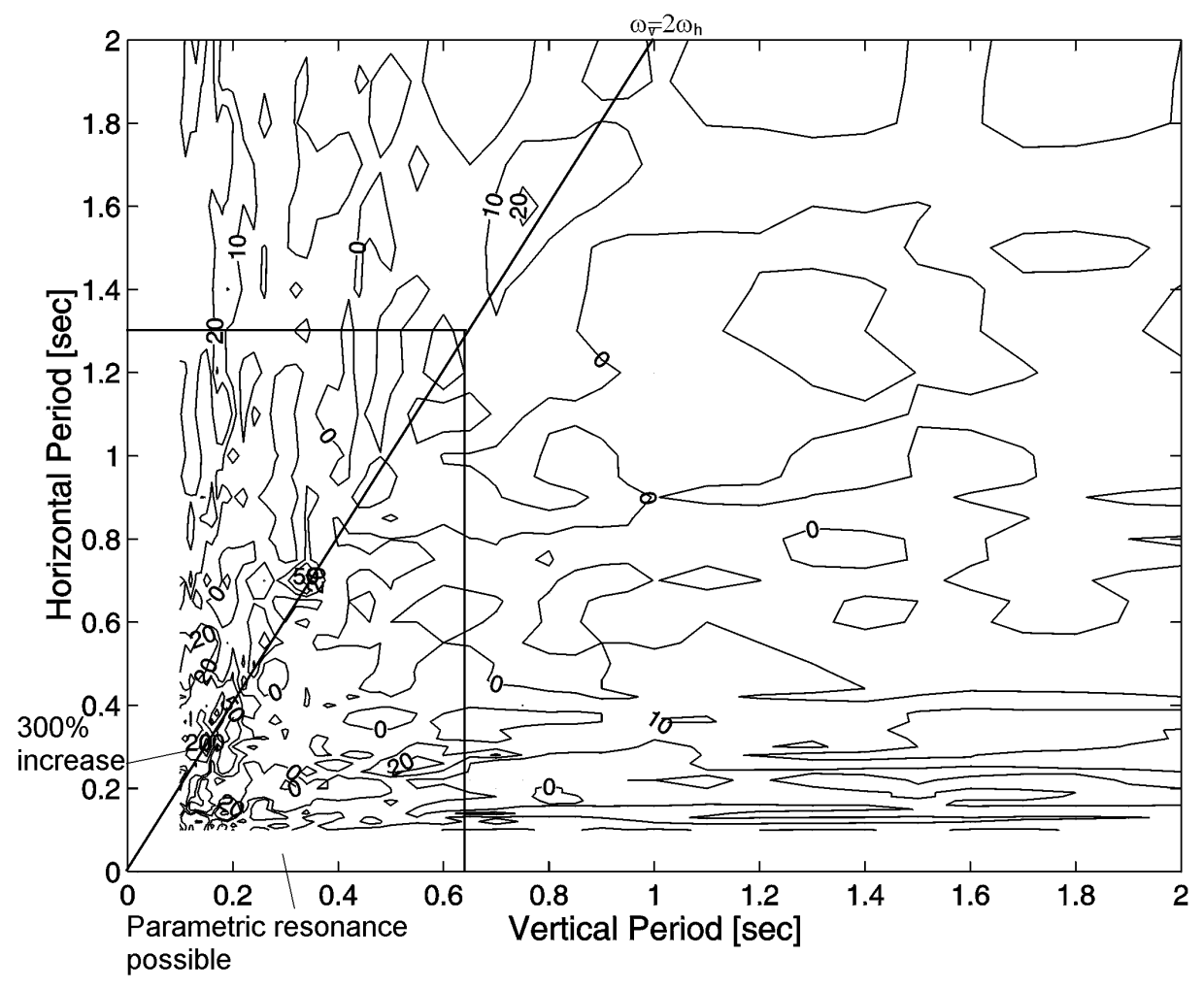

Figure 17: 


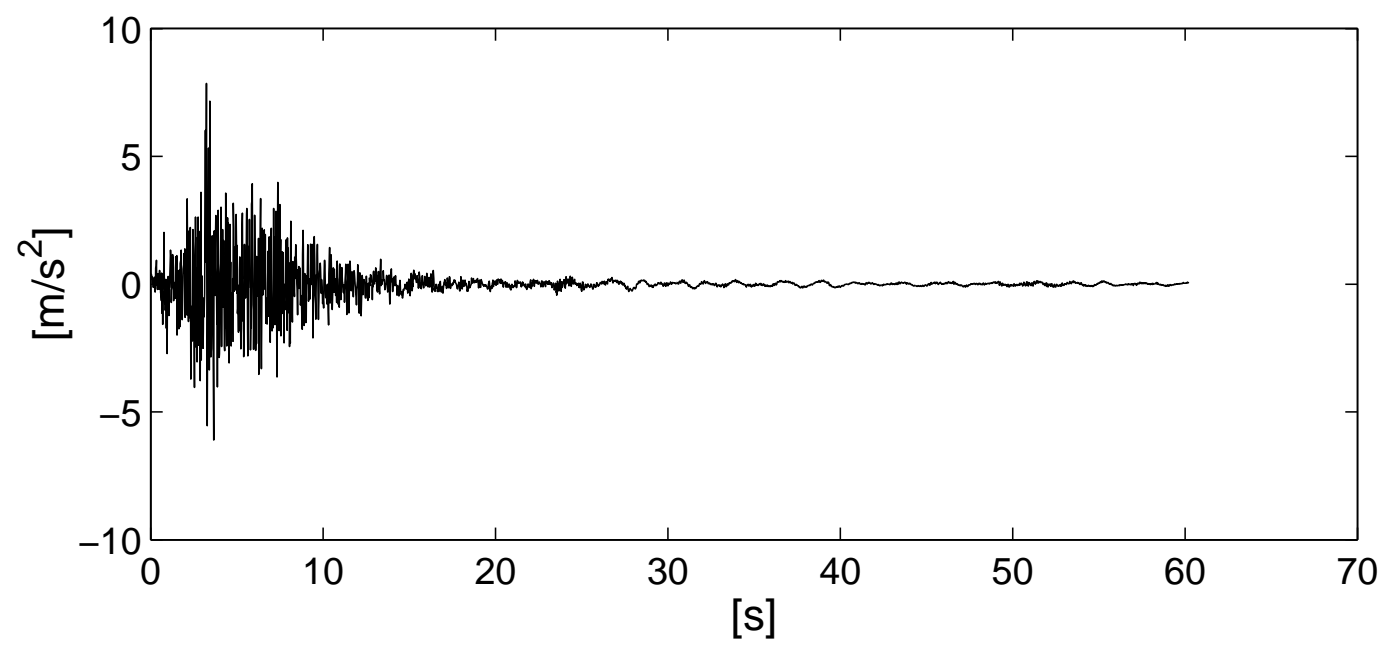

(a)

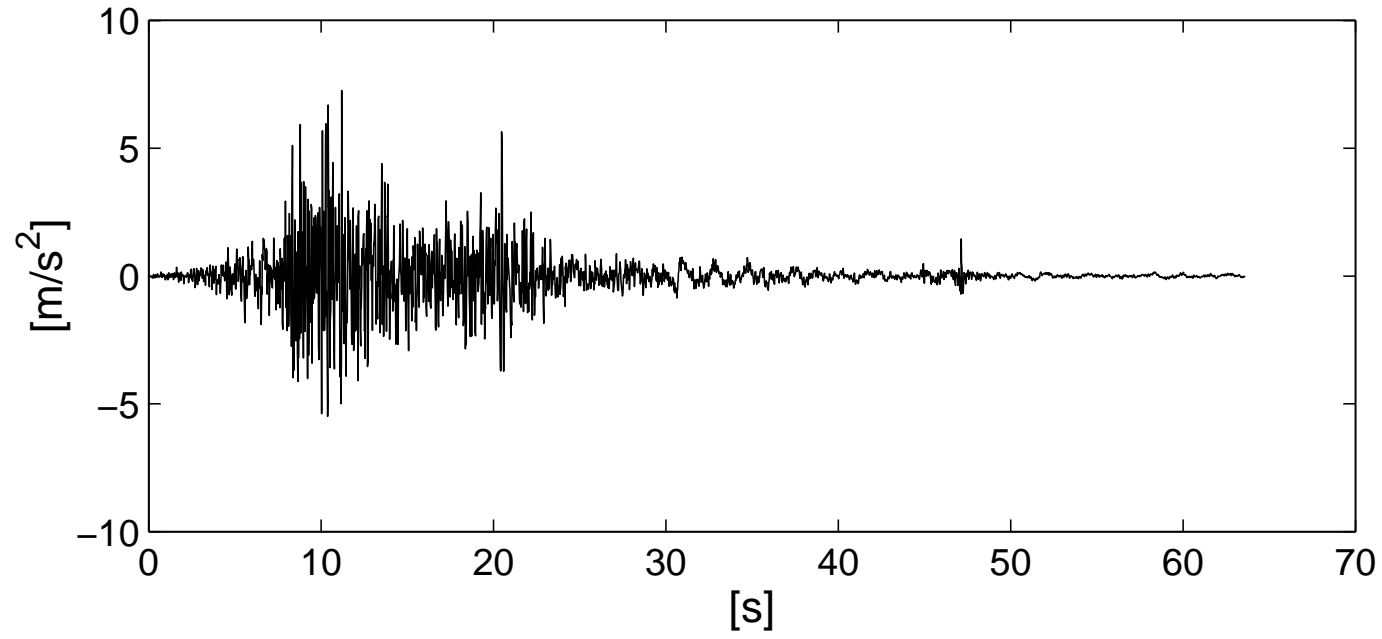

(b)

Figure 18: 


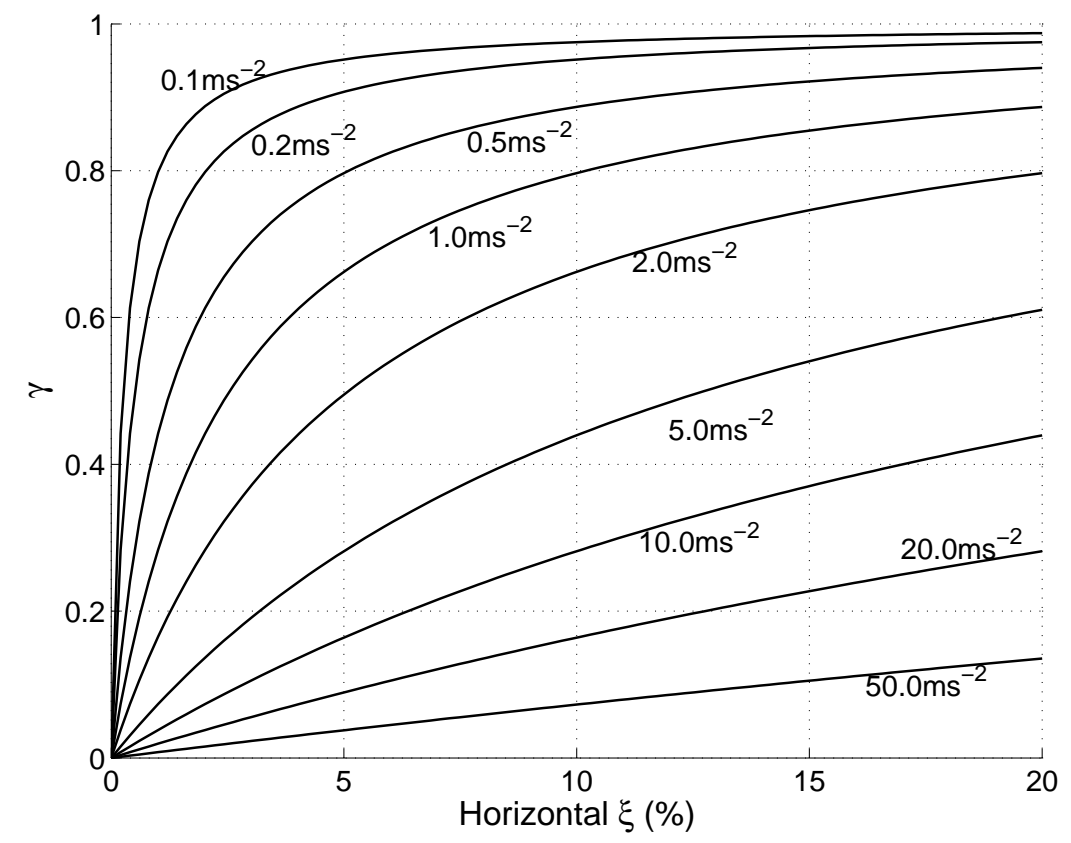

Figure 19:

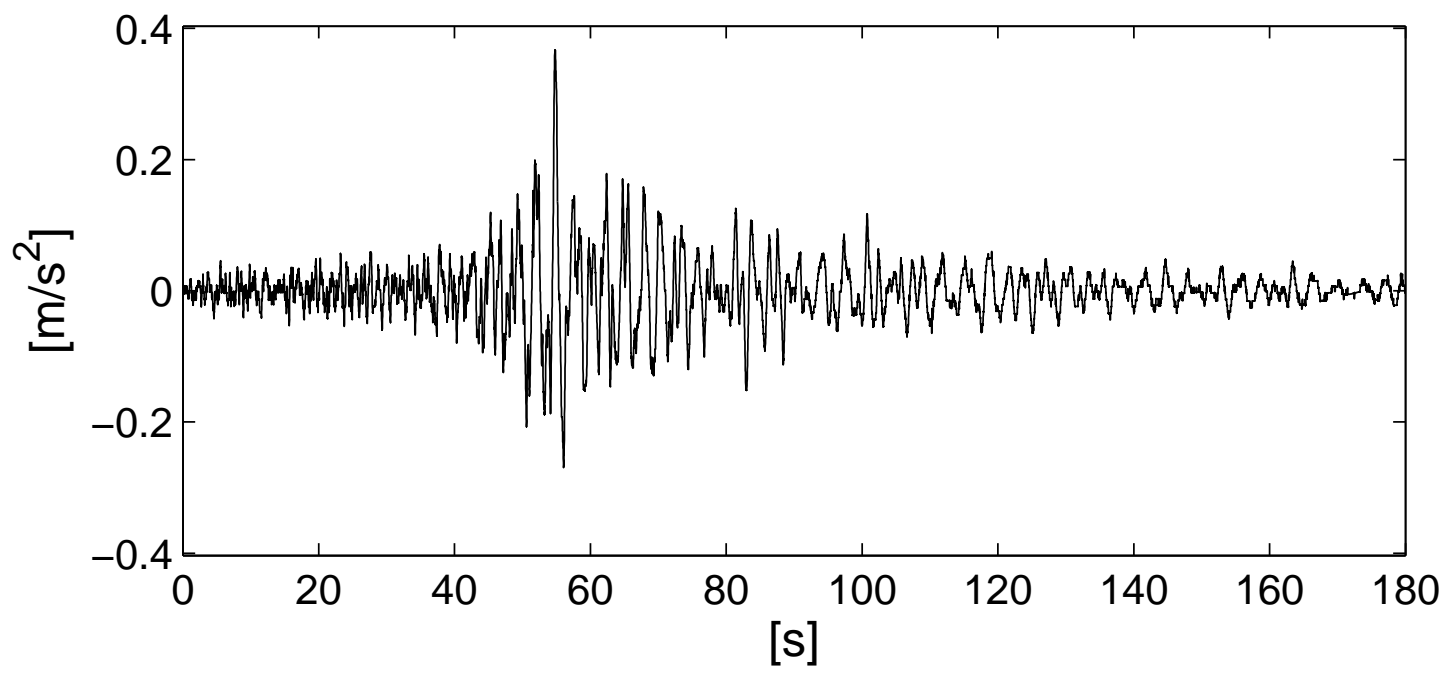

Figure 20: 


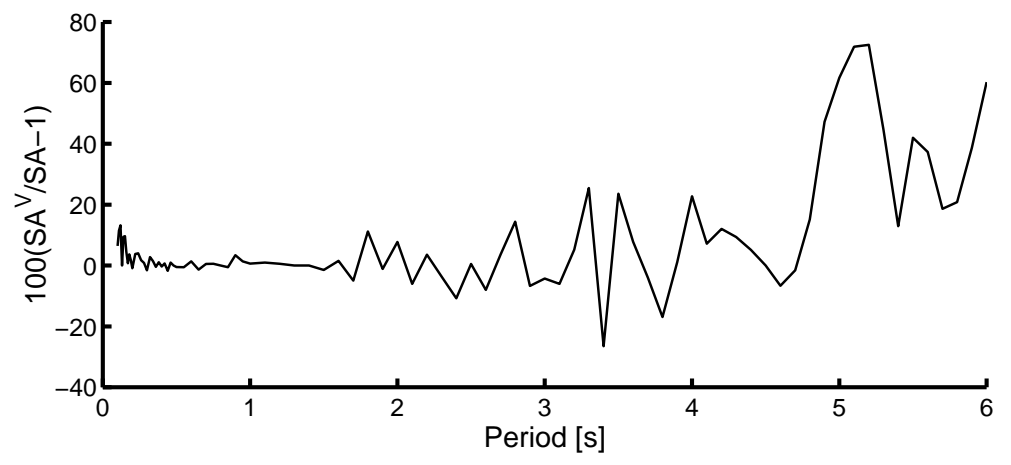

Figure 21:

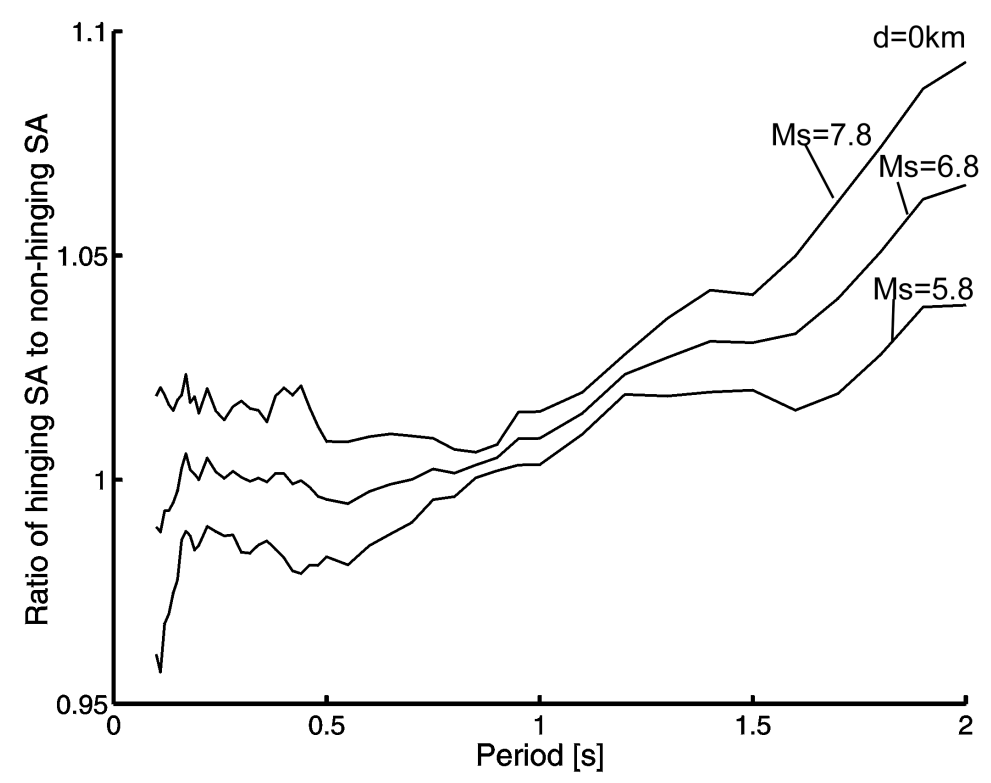

Figure 22: 


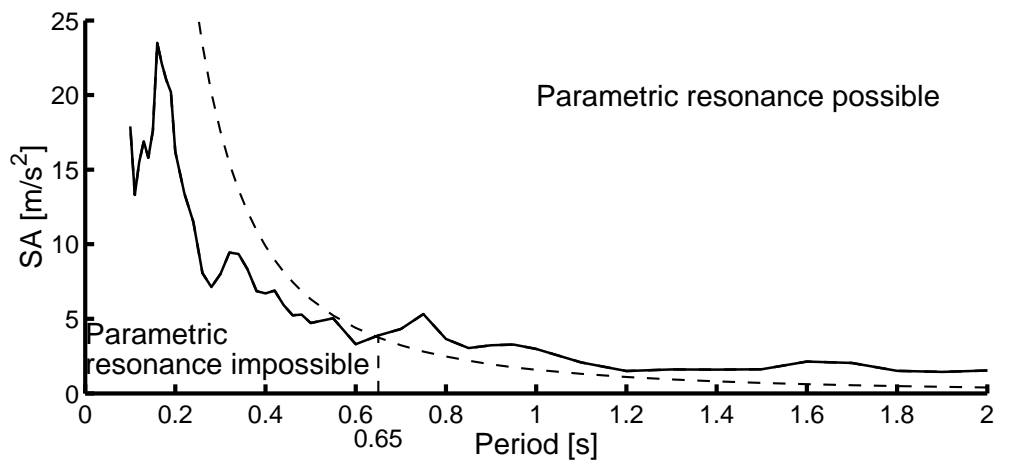

Figure 23:

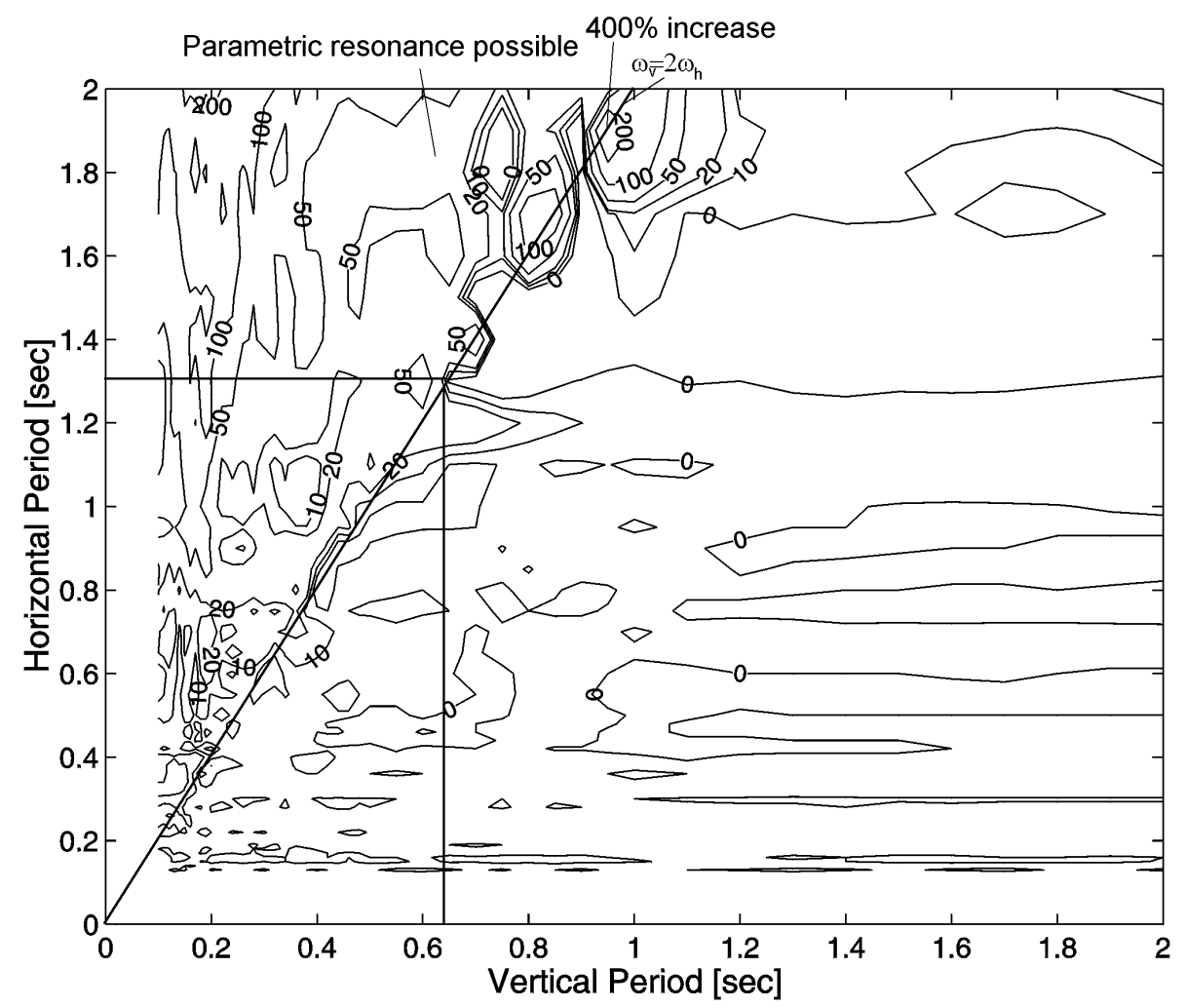

Figure 24: 


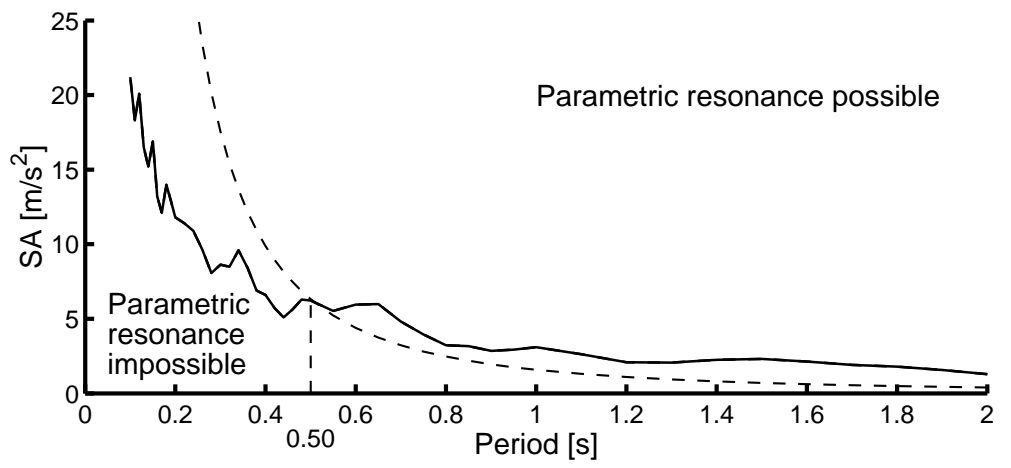

Figure 25:

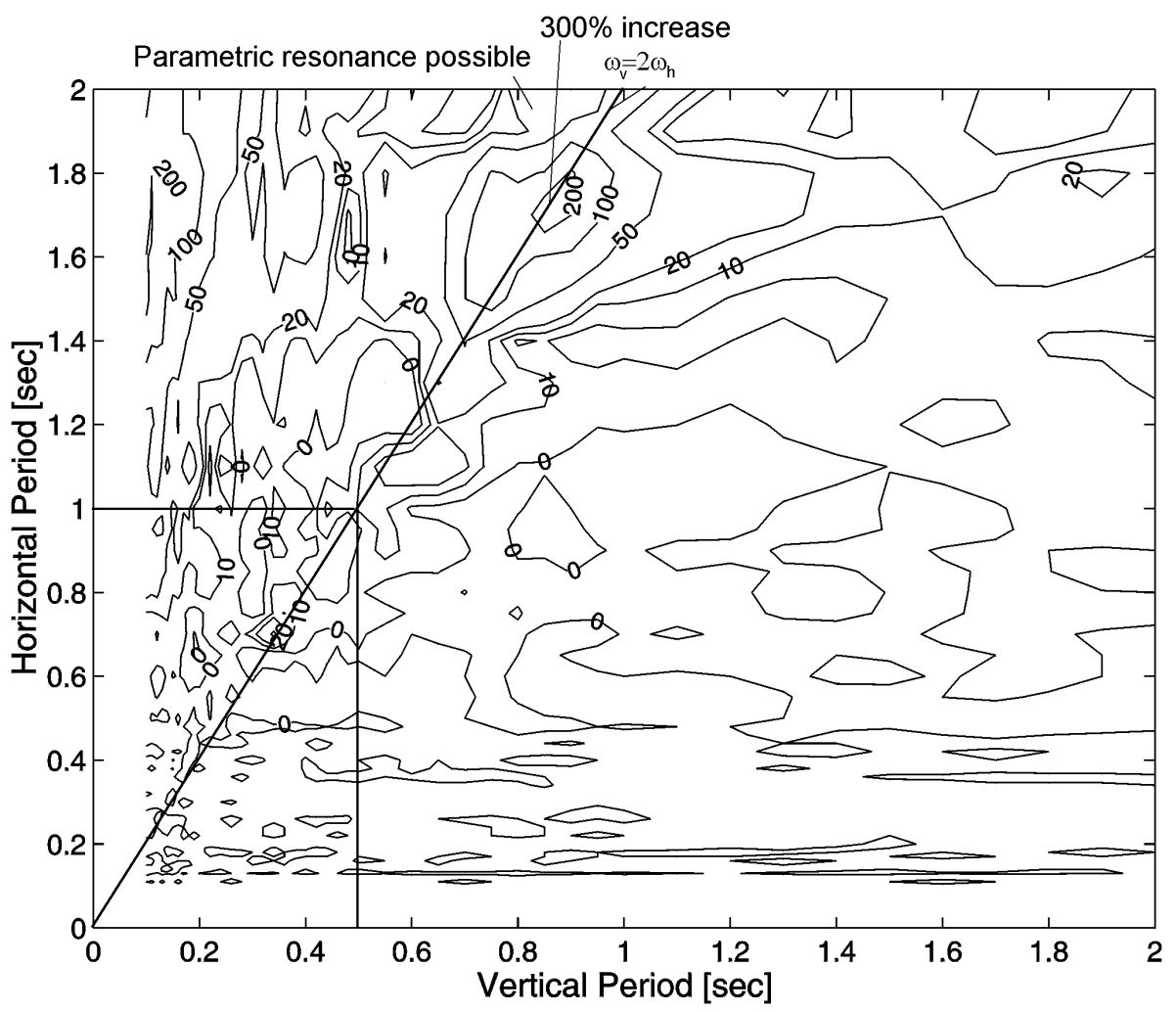

Figure 26: 


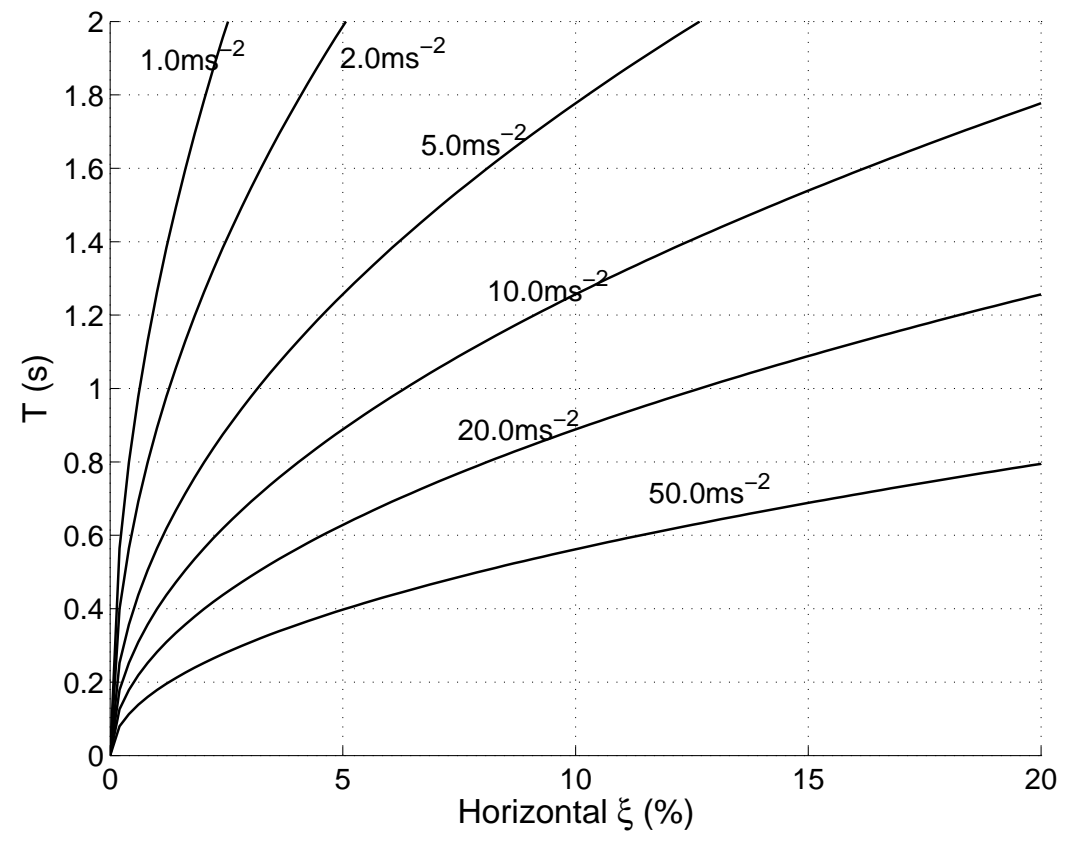

(a)

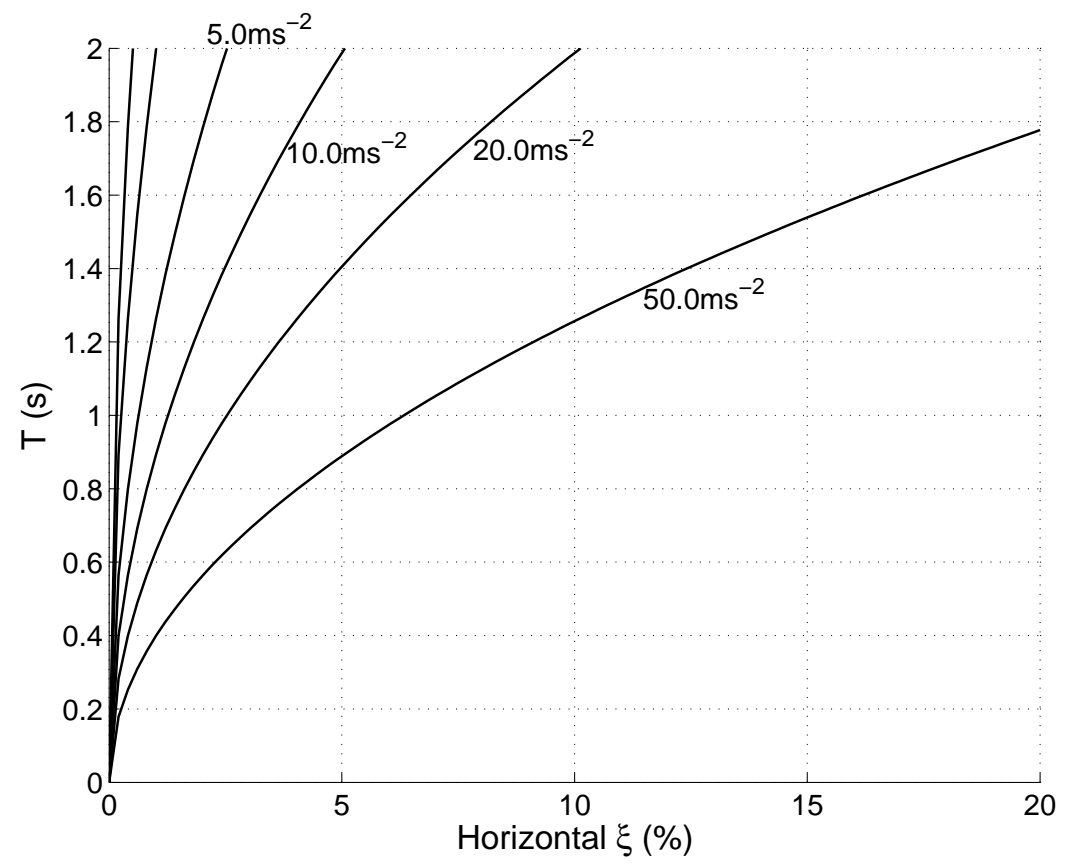

(b)

Figure 27: 


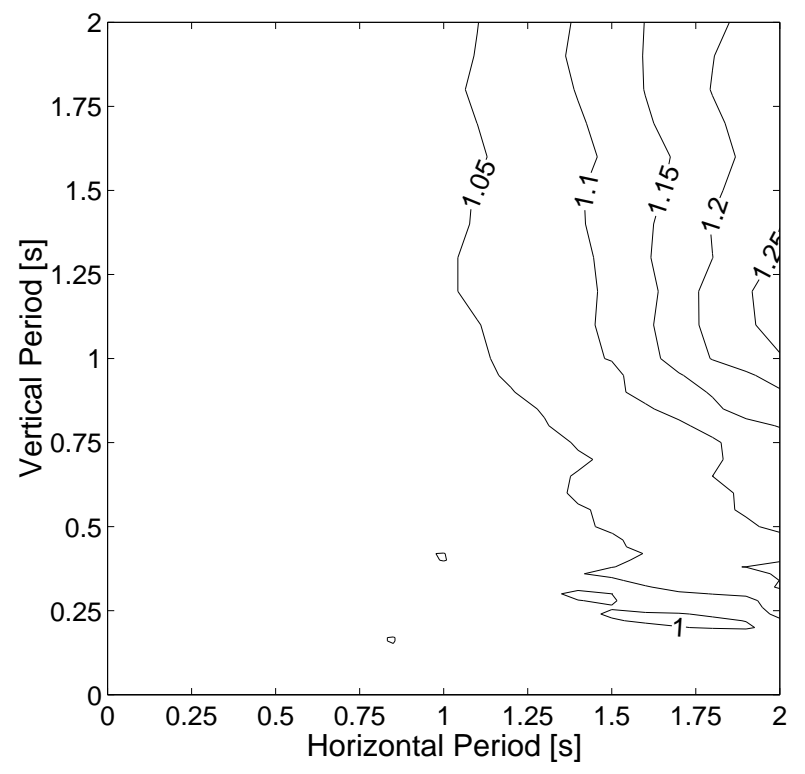

Figure 28: 